\title{
The recovery of coral genetic diversity in the Sunda Strait following the 1883 eruption of Krakatau
}

\author{
C. J. Starger - P. H. Barber - Ambariyanto $\cdot$ \\ A. C. Baker
}

Received: 28 September 2009/Accepted: 19 February 2010/Published online: 11 March 2010

(C) The Author(s) 2010. This article is published with open access at Springerlink.com

\begin{abstract}
Surveys of microsatellite variation show that genetic diversity has largely recovered in two reef-building corals, Pocillopora damicornis and Seriatopora hystrix (Scleractinia: Pocilloporidae), on reefs which were decimated by the eruption of the volcano Krakatau in 1883 . Assignment methods and gene flow estimates indicate that the recolonization of Krakatau occurred mainly from the closest upstream reef system, Pulau Seribu, but that larval
\end{abstract}

Communicated by Biology Editor Dr. Ruth Gates

\section{J. Starger}

Department of Ecology, Evolution, and Environmental Biology, Columbia University, MC-5557, 1200 Amsterdam Avenue,

New York, NY 10027, USA

\section{J. Starger}

Sackler Institute for Comparative Genomics, American Museum of Natural History, 79th Street and Central Park West,

New York, NY 10024, USA

C. J. Starger $(\bowtie) \cdot$ P. H. Barber

Department of Ecology and Evolutionary Biology, University of California Los Angeles, 621 Charles Young South Drive,

Los Angeles, CA 90024, USA

e-mail: cstarger@ucla.edu

\section{Ambariyanto}

Faculty of Fisheries and Marine Sciences, Diponegoro

University, Kampus Tembalang, Semarang, Indonesia

\section{A. C. Baker}

Division of Marine Biology and Fisheries, Rosenstiel School of Marine and Atmospheric Science, University of Miami, 4600 Rickenbacker Causeway, Miami, FL 33149, USA

A. C. Baker

Wildlife Conservation Society, Marine Program,

2300 Southern Blvd., Bronx, NY 10460, USA input from other regions has also occurred. This pattern is clearer in $S$. hystrix, which is traditionally the more dispersal-limited species. Despite these observed patterns of larval dispersal, self-recruitment appears to now be the most important factor in supplying larvae to coral populations in Krakatau. This suggests that the colonization of devastated reefs can occur quickly through larval dispersal; however, their survival requires local sources of larvae for self-recruitment. This research supports the observation that the recovery of genetic diversity in coral reef animals can occur on the order of decades and centuries rather than millennia. Conservation measures aimed at sustaining coral reef populations in Krakatau and elsewhere should include both the protection of upstream source populations for larval replenishment should disaster occur as well as the protection of large adult colonies to serve as local larval sources.

Keywords Dispersal $\cdot$ Recovery $\cdot$ Pocillopora . Seriatopora $\cdot$ Microsatellite $\cdot$ Volcano

\section{Introduction}

On August 26, 1883, the eruption and near-total destruction of the volcano Krakatau in the Sunda Strait, Indonesia, completely exterminated all marine life in the surrounding area. Pyroclastic flows deposited molten rock and ash at a temperature of $475-550^{\circ} \mathrm{C}$ to an average thickness of $20 \mathrm{~m}$ (Mandeville et al. 1994) on the surrounding sea floor (Sigurdsson et al. 1991). It is the scientific consensus that all life within a $15 \mathrm{~km}$ radius was completely extinguished by this eruption (Simkin and Fiske 1983; Thornton 1996). A new volcanic island Anak Krakatau ("the Child of Krakatau") has been rising in the caldera since August 
1930 and is now approximately $450 \mathrm{~m}$ high. Fringing coral reefs have subsequently formed on Anak Krakatau, the remnant islands of Krakatau, and the surrounding areas that were affected by the eruption and resulting tsunami, presenting researchers with a unique opportunity to study the assembly and development of a benthic ecosystem following its complete destruction.

The recovery of terrestrial communities on Krakatau is well documented (Simkin and Fiske 1983; Thornton 1996). However, its marine ecosystems have been largely ignored until recent years (review: Barber et al. 2002). Sluiter (1890) observed coral recruitment on Krakatau less than a decade following the eruption. However, this early reef was subsequently smothered by further volcanic activity (Umbgrove 1930). It is unknown at what point contemporary coral reefs took hold in the region or which reefs served as source populations for the colonization of the Krakatau region. The assembly and structure of coral communities in general has been thoroughly studied (review: Karlson 2002). However, their recruitment and ecological succession on volcanoes has only been investigated in a few cases. Grigg and Maragos (1974) observed coral settlers on lava that was less than 2 years old in Hawaii. Tomascik et al. (1996) report an exceptionally diverse coral community on 5-year-old lava following a major eruption of Gunung Api in the Banda Sea, Eastern Indonesia, forming what may be a new refuge and larval source for surrounding areas. However, colonization in these cases may have come from local sources directly adjacent to the lava flows in question. Due to the extent of destruction in Krakatau, all colonization must have come from elsewhere.

With no additional examples of de novo reef formation, it is difficult to predict how corals would have first colonized Krakatau; where they would have come from, or how ecological succession would have proceeded. In the only contemporary research done on the coral reefs of Krakatau, Barber et al. (2002) observed the rapid recovery of genetic diversity in the stomatopod crustaceans Haptosquilla pulchella and $H$. glyptocercus. Larval sources for Krakatau were restricted to coral reefs south of the Java and Flores Seas, a putative barrier to marine larval dispersal (Barber et al. 2000).

In the study presented here, the genetic consequences of recolonization were examined in two common Indo-Pacific reef-building stony corals, Pocillopora damicornis and Seriatopora hystrix. These corals occupy similar reef habitats, yet differ notably in their population genetic structures. $P$. damicornis typically displays more genetically open populations with high gene flow, whereas S. hystrix populations are often more closed, exhibiting higher levels of genetic subdivision (Ayre and Dufty 1994; Ayre et al. 1997; Ayre and Hughes 2000, 2004). Although both species brood larvae on a lunar cycle throughout the year in Australia, Japan, the Philippines, and Taiwan (Atoda 1947, 1951; Harrison and Wallace 1990; Fan et al. 2006; Villanueva et al. 2008), P. damicornis is known to brood asexual larvae in Western Australia and Hawai'i (Stoddart 1984) whereas S. hystrix broods are usually generated sexually (Ayre and Resing 1986; Sherman 2008). However, P. damicornis recruits on eastern Australian reefs appear to be predominantly sexually generated, and so may have been derived from broadcast spawning (Ayre and Miller 2004; Miller and Ayre 2008) a phenomenon also observed in $P$. damicornis in Eastern Pacific Panamá (Glynn et al. 1991).

Overall, these differences indicate a complex relationship between reproduction and genetic structure, but generally suggest higher effective dispersal in $P$. damicornis than S. hystrix. As such, $P$. damicornis populations from Krakatau are hypothesized to recover genetic diversity more rapidly than S. hystrix and larval sources will be more varied. Support for this hypothesis would be evident in the identification of multiple source populations for P. damicornis, high gene flow estimates from areas outside of Krakatau, and gene diversity values that are similar to other, older regions in Indonesia. By contrast, source populations for S. hystrix in Krakatau would most likely be restricted to those immediately upstream (from reefs in the Java Sea). Gene flow estimates should also be lower in this species, indicative of a slower recovery of genetic diversity on Krakatau.

\section{Methods}

\section{Fieldwork}

Coral samples were collected by scuba and snorkeling in 2005 and 2006 (Table 1). In total, 682 samples of $P$. damicornis and 823 samples of S. hystrix were collected from across the Indonesian Archipelago for comparative analysis to a subset from the Krakatau region, 122 and 197 samples, respectively (Fig. 1). One branch was removed from each adult colony to ensure nonlethal sampling and minimal impact on the individual. Because reproductive maturity depends on colony size rather than age (Hughes 1984; Lirman 2000; Zakai et al. 2000), adults were defined as colonies greater than $7 \mathrm{~cm}$ in diameter for $P$. damicornis and $8 \mathrm{~cm}$ for $S$. hystrix (Harrison and Wallace 1990). Species identifications followed Veron (2000). Samples were taken from colonies at least $10 \mathrm{~m}$ apart to help minimize resampling of genetic clonemates that may have resulted from fragmentation. 
Table 1 Sample regions and localities listed in approximate east to west order

\begin{tabular}{|c|c|c|c|c|c|}
\hline Region & Locality & Latitude & Longitude & P. damicornis & S. hystrix \\
\hline Aceh & Pulau Weh & $5^{\circ} 36.99 \mathrm{~N}$ & $95^{\circ} 41.980 \mathrm{E}$ & 22 & 25 \\
\hline \multirow[t]{9}{*}{ Krakatau } & Anak Krakatau & $6^{\circ} 05.43 \mathrm{~S}$ & $105^{\circ} 25.10 \mathrm{E}$ & 19 & 22 \\
\hline & Anyer & $6^{\circ} 04.61 \mathrm{~S}$ & $105^{\circ} 52.90 \mathrm{E}$ & 21 & 0 \\
\hline & Karang Serang & $6^{\circ} 06.57 \mathrm{~S}$ & $105^{\circ} 26.39 \mathrm{E}$ & 17 & 0 \\
\hline & Rakata (Krakatau Besar) & $6^{\circ} 08.83 \mathrm{~S}$ & $105^{\circ} 27.80 \mathrm{E}$ & 26 & 18 \\
\hline & Rakata Kecil & $6^{\circ} 05.86 \mathrm{~S}$ & $105^{\circ} 27.07 \mathrm{E}$ & 24 & 0 \\
\hline & Sangiang & $5^{\circ} 57.80 \mathrm{~S}$ & $105^{\circ} 51.88 \mathrm{E}$ & 25 & 24 \\
\hline & Sebesi & $5^{\circ} 55.80 \mathrm{~S}$ & $105^{\circ} 30.93 \mathrm{E}$ & 22 & 9 \\
\hline & Sebuku 1 & $5^{\circ} 52.78 \mathrm{~S}$ & $105^{\circ} 32.03 \mathrm{E}$ & 20 & 24 \\
\hline & Sebuku 2 & $5^{\circ} 53.49 \mathrm{~S}$ & $105^{\circ} 30.13 \mathrm{E}$ & 23 & 25 \\
\hline \multirow[t]{6}{*}{ Pulau Seribu } & Alam Kotok & $5^{\circ} 41.98 \mathrm{~S}$ & $106^{\circ} 32.29 \mathrm{E}$ & 22 & 20 \\
\hline & Belat & $5^{\circ} 37.70 \mathrm{~S}$ & $106^{\circ} 34.38 \mathrm{E}$ & 20 & 21 \\
\hline & Karang Congkak & $5^{\circ} 42.53 \mathrm{~S}$ & $106^{\circ} 34.34 \mathrm{E}$ & 18 & 21 \\
\hline & Pramuka & $5^{\circ} 44.77 \mathrm{~S}$ & $106^{\circ} 35.51 \mathrm{E}$ & 10 & 23 \\
\hline & Pulau Pari & $5^{\circ} 50.00 \mathrm{~S}$ & $106^{\circ} 36.00 \mathrm{E}$ & 13 & 38 \\
\hline & Semak Daun & $5^{\circ} 44.33 \mathrm{~S}$ & $106^{\circ} 33.83 \mathrm{E}$ & 23 & 21 \\
\hline \multirow[t]{3}{*}{ Bali } & Napoleon Reef & $8^{\circ} 07.57 \mathrm{~S}$ & $114^{\circ} 38.13 \mathrm{E}$ & 0 & 21 \\
\hline & Seraya & $8^{\circ} 16.57 \mathrm{~S}$ & $115^{\circ} 35.72 \mathrm{E}$ & 21 & 20 \\
\hline & Pemuteran & $8^{\circ} 07.26 \mathrm{~S}$ & $114^{\circ} 37.53 \mathrm{E}$ & 11 & 41 \\
\hline Lombok & Gili Trawangan & $6^{\circ} 05.52 \mathrm{~S}$ & $120^{\circ} 24.83 \mathrm{E}$ & 18 & 12 \\
\hline \multirow[t]{2}{*}{ Makassar } & Bone Batang & $5^{\circ} 02.10 \mathrm{~S}$ & $119^{\circ} 16.33 \mathrm{E}$ & 0 & 24 \\
\hline & Barrang Lompo & $5^{\circ} 02.70 \mathrm{~S}$ & $119^{\circ} 19.27 \mathrm{E}$ & 30 & 0 \\
\hline Selayar & Gusung & $6^{\circ} 05.52 \mathrm{~S}$ & $120^{\circ} 24.83 \mathrm{E}$ & 0 & 23 \\
\hline \multirow[t]{2}{*}{ Flores } & Kukusan & $8^{\circ} 32.96 \mathrm{~S}$ & $119^{\circ} 48.25 \mathrm{E}$ & 0 & 1 \\
\hline & Sebayur & $8^{\circ} 30.44 \mathrm{~S}$ & $119^{\circ} 42.67 \mathrm{E}$ & 0 & 17 \\
\hline \multirow[t]{5}{*}{ Northern Sulawesi } & Batu Gosok (Bangka) & $1^{\circ} 47.59 \mathrm{~N}$ & $125^{\circ} 11.18 \mathrm{E}$ & 0 & 12 \\
\hline & Murex House Reef (Bangka) & $1^{\circ} 44.15 \mathrm{~N}$ & $125^{\circ} 08.95 \mathrm{E}$ & 2 & 34 \\
\hline & Nudi Retreat (Lembeh) & $1^{\circ} 29.00 \mathrm{~N}$ & $125^{\circ} 14.41 \mathrm{E}$ & 26 & 36 \\
\hline & Pisok (Manado) & $1^{\circ} 34.43 \mathrm{~N}$ & $124^{\circ} 48.17 \mathrm{E}$ & 25 & 5 \\
\hline & Manado Tua & $1^{\circ} 36.99 \mathrm{~N}$ & $124^{\circ} 41.68 \mathrm{E}$ & 2 & 0 \\
\hline \multirow[t]{4}{*}{ Halmahera } & Doi & $2^{\circ} 16.60 \mathrm{~N}$ & $127^{\circ} 46.79 \mathrm{E}$ & 0 & 20 \\
\hline & Jerewai & $1^{\circ} 31.23 \mathrm{~N}$ & $128^{\circ} 42.02 \mathrm{E}$ & 17 & 22 \\
\hline & Tidore & $0^{\circ} 45.21 \mathrm{~N}$ & $127^{\circ} 24.56 \mathrm{E}$ & 11 & 28 \\
\hline & Tonuu & $1^{\circ} 47.91 \mathrm{~N}$ & $127^{\circ} 59.97 \mathrm{E}$ & 21 & 20 \\
\hline \multirow[t]{6}{*}{ Raja Ampat } & Alyui & $0^{\circ} 10.47 \mathrm{~S}$ & $130^{\circ} 14.85 \mathrm{E}$ & 20 & 19 \\
\hline & Gam & $0^{\circ} 25.88 \mathrm{~S}$ & $130^{\circ} 33.16 \mathrm{E}$ & 4 & 1 \\
\hline & Jefman & $0^{\circ} 55.64 \mathrm{~S}$ & $131^{\circ} 07.41 \mathrm{E}$ & 22 & 22 \\
\hline & Kri Island & $0^{\circ} 33.38 \mathrm{~S}$ & $130^{\circ} 40.68 \mathrm{E}$ & 30 & 9 \\
\hline & Manta Point & $0^{\circ} 33.71 \mathrm{~S}$ & $130^{\circ} 32.42 \mathrm{E}$ & 1 & 0 \\
\hline & Mayalibit & $0^{\circ} 17.85 \mathrm{~S}$ & $130^{\circ} 48.49 \mathrm{E}$ & 9 & 20 \\
\hline \multirow[t]{3}{*}{ Biak } & Adoki & $1^{\circ} 08.53 \mathrm{~S}$ & $135^{\circ} 59.68 \mathrm{E}$ & 10 & 12 \\
\hline & Owi & $1^{\circ} 15.26 \mathrm{~S}$ & $136^{\circ} 10.99 \mathrm{E}$ & 9 & 9 \\
\hline & Rasbar & $1^{\circ} 15.28 \mathrm{~S}$ & $136^{\circ} 19.07 \mathrm{E}$ & 0 & 4 \\
\hline \multirow[t]{2}{*}{ Yapen } & Ambai & $1^{\circ} 57.64 \mathrm{~S}$ & $136^{\circ} 19.23 \mathrm{E}$ & 15 & 22 \\
\hline & Serui & $1^{\circ} 54.32 \mathrm{~S}$ & $136^{\circ} 13.65 \mathrm{E}$ & 6 & 0 \\
\hline Manokwari & Lemon & $0^{\circ} 53.41 \mathrm{~S}$ & $134^{\circ} 04.90 \mathrm{E}$ & 18 & 20 \\
\hline Teluk Cenderwasih & Rumberpon & $1^{\circ} 44.23 \mathrm{~S}$ & $134^{\circ} 12.15 \mathrm{E}$ & 13 & 19 \\
\hline
\end{tabular}


Table 1 continued

\begin{tabular}{llllrr}
\hline Region & Locality & Latitude & Longitude & P. damicornis & S. hystrix \\
\hline Kaimana & Mauwara & $3^{\circ} 49.65 \mathrm{~S}$ & $134^{\circ} 03.51 \mathrm{E}$ & 23 & 0 \\
& Namatote & $3^{\circ} 46.73 \mathrm{~S}$ & $133^{\circ} 52.93 \mathrm{E}$ & 0 & 21 \\
Fakfak & Momon & $3^{\circ} 56.38 \mathrm{~S}$ & $132^{\circ} 48.21 \mathrm{E}$ & 4 & 18 \\
\hline
\end{tabular}

Fig. 1 Study localities in Indonesia with close-up view of the localities in the Krakatau region
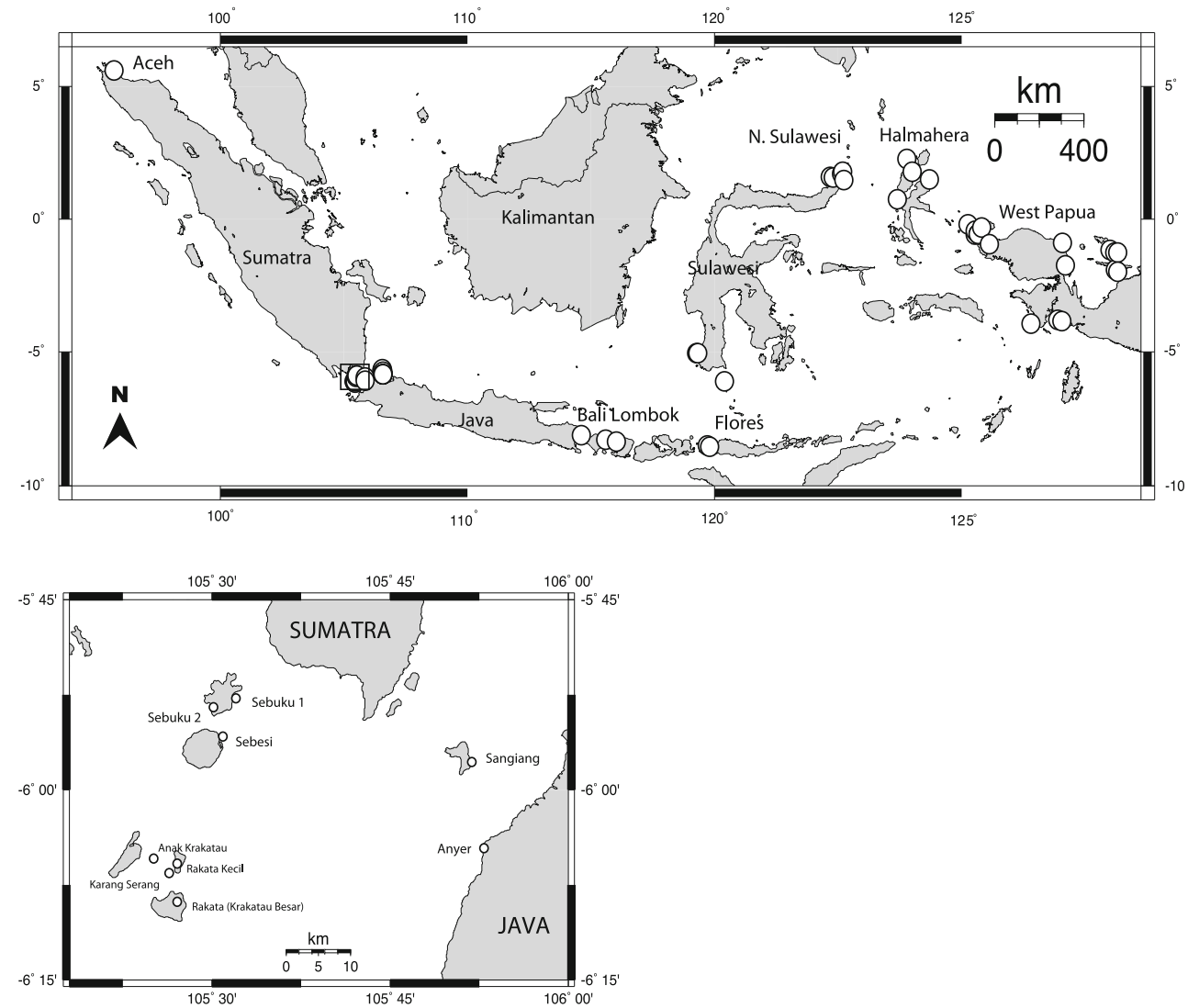

Genetic analysis

Genomic DNA was extracted using a modified Chelex protocol (Walsh et al. 1991) in which tissue from 1 or 2 polyps was lysed at $95^{\circ} \mathrm{C}$ for $1 \mathrm{~h}$ in $300 \mu \mathrm{l}$ of $5 \%$ Chelex. The resulting solution was briefly vortexed and centrifuged at $13,000 \mathrm{~g}$ for $1 \mathrm{~min}$. The supernatant was then used as the template for the polymerase chain reaction (PCR). $P$. damicornis PCR for 6 microsatellite markers, $\mathrm{Pd} 2-001$ through Pd2-006, followed the protocol of Starger et al. (2008). PCR conditions for PV2, PV6, and PV7 followed the protocol of Magalon et al. (2004). Previously published markers Sh2-005, Sh2-006, Sh3-003, Sh3-004, Sh3-008, Sh4-001, and Sh4-010 were amplified in S. hystrix samples following the PCR procedure of Underwood et al. (2006). Size fragment analysis for both species was performed with fluorescent-labeled primers on an ABI 3730xl running GeneMapper 3.5 software. Alleles were coded as the number of microsatellite repeats.
Genetic diversity

Clonal diversity could not be statistically assessed due to the need for an explicit, transect-based sampling strategy (Arnaud-Haond et al. 2007) that was not possible in this case due to time constraints in the field. However, in order to avoid any spurious results that might come from sampling genetic clones, all but one representative of each multilocus genotype was removed from the dataset before further analysis. Hardy-Weinberg equilibrium (HWE) was assessed by estimating $F_{\text {Is }}$ for each locus in each population in GenoDive 2.0b16 (Meirmans and Van Tienderen 2004). Significantly positive $F_{\text {IS }}$ values indicate heterozygote excess whereas negative values indicate heterozygote deficit. Statistical significance was tested with 100,000 permutations at $p=0.05$ and the false discovery rate (FDR) correction for multiple test (Benjamini and Hochberg 1995). Observed and expected heterozygosities $\left(\mathrm{H}_{\mathrm{o}}\right.$ and $\mathrm{H}_{\mathrm{e}}$ ) were calculated in GenAlEx 6.3 (Peakall and 
Smouse 2006). The possible presence of null alleles was investigated in Genepop on the Web (Raymond and Rousset 1995). Deviations from linkage equilibrium were assessed with likelihood ratio tests with a significance level of $p=0.05$ (Slatkin and Excoffier 1996) as implemented in Arlequin 3.11 (Excoffier et al. 2005).

In order to compare the genetic diversity found in Krakatau to older reefs throughout Indonesia, Nei's unbiased genetic diversity (Hz) (Nei 1987) was calculated for each locality using the Excel Microsatellite Toolkit (Park 2001). Diversity comparisons between species were made using the Wilcoxon Signed Rank Test. The mean ratio of the number of alleles to the range in allele size $(M)$ was calculated for each locality in Arlequin 3.11 to detect recent reductions in population size (Garza and Williamson 2001) that might be associated with the decimation and recolonization of the islands of Krakatau.

\section{Assignment methods}

To examine the potential source populations for recolonization of Krakatau spatial and non-spatial assignment methods were implemented in BAPS 5.3 (Corander and Marttinen 2006; Corander et al. 2008). Populations with fewer than fifteen genetically distinct individuals were excluded from this analysis. BAPS implements a stochastic optimization procedure rather than a Markov chain Monte Carlo (MCMC), and therefore generally performs more quickly than the program Structure (Falush et al. 2003) with similar results (Latch et al. 2006). Individuals were grouped into the localities from which they were sampled using the 'cluster by groups' option, and structure was inferred as clusters of groups. This method was employed because the distance between individuals within localities is trivial relative to the scale of the entire study. The 'cluster by groups' option was therefore most appropriate (see Corander et al. 2008 for a detailed description). In each analysis, maximum $K$ (the number of genetic clusters) was input at intervals of 5 from 5 to 50 (program input: 5 , $10,15,20,25,30,35,40,45,50)$. The $K$ value with the lowest $\log$ likelihood, " $\log (\mathrm{ml})$ ", was then called $K^{\prime}$ and a more focused search was run a further 5 times with maximum $K$ set to $K^{\prime}, K^{\prime}-1$ and $K^{\prime}+1$ (example input for $K^{\prime}=15: 14,14,14,14,14,15,15,15,15,15,16,16,16$, $16,16)$. From these results, the $K$ value with the lowest log (ml), called $K$, was used to chart the genetic clustering of localities. The spatial method incorporates the geographic proximity of samples into the assignment algorithm when the genetic data are insufficient to resolve cluster membership. Likelihood scores between the spatial and non-spatial methods are directly comparable within each species and are informative as to which analysis produces the more likely structure (J. Corander, pers. comm.). The conditional posterior probability, or 'local uncertainty,' in the assignment of a locality to a specific cluster was also calculated by BAPS (equation 11 in Corander et al. 2008). Admixture inferences were not used because of a strong need for a biologically meaningful number of ancestral populations which is used as prior information (Corander and Marttinen 2006) and which was not available in this case.

\section{Genetic structure and migration}

In order to infer genetic differentiation among regions, with the aim of identifying source populations for the recolonization of the Sunda Strait, pairwise $F_{\mathrm{ST}}$ values were calculated in Arlequin 3.11 and tested for significance at $p=0.05$ with the FDR correction for multiple test (Benjamini and Hochberg 1995).

A matrix of pairwise immigration was also estimated among regions using the Bayesian assignment method implemented in BayesAss +1.2 (Wilson and Rannala 2003). Default settings for burn-in (200,000), number of MCMC iterations (3 million), and sampling frequency $(2,000)$ were appropriate to reach convergence based on visual inspection of likelihood scores. Regions with fewer than fifteen genetically distinct individuals were excluded from this analysis.

\section{Results}

Heterozygosity, clonality, and genetic diversity

For $P$. damicornis, 682 individuals were genotyped and analyzed at 9 microsatellite loci. The number of alleles per locus ranged from 8 at locus Pd2-006 to 28 at locus PV2 (mean $=14.00)$. Twelve multilocus genotypes were observed in more than one individual; however, only four of these were observed in more than two individuals (Table 4). In total, $14 P$. damicornis individuals were removed from subsequent analyses.

For S. hystrix, 823 individuals were genotyped and analyzed at 7 microsatellite loci. The number of alleles per locus ranged from 8 at locus Sh3-003 and Sh3-008 to 24 at locus Sh2-006 (mean = 14.43). Forty-nine multilocus genotypes were observed in more than one individual; however, only 12 of these were observed in more than two individuals (Table 5). In total, 74 S. hystrix individuals were removed from subsequent analyses.

Departures from Hardy-Weinberg equilibrium were evident in many cases based on significantly positive and negative $F_{\text {IS }}$ values (Tables 2 and 3 ) and, as a result, Genepop detected the possibility of null alleles in many populations (data not shown). Similarly, linkage 
Table 2 Allele data for $P$. damicornis for populations where $\mathrm{Ng} \geq 15$

\begin{tabular}{|c|c|c|c|c|c|c|c|c|c|c|}
\hline & & $\mathrm{Pd} 2-001$ & $\mathrm{Pd} 3-002$ & $\mathrm{Pd} 2-003$ & $\mathrm{Pd} 3-004$ & Pd3-005 & $\mathrm{Pd} 2-006$ & Pv2 & Pv6 & Pv7 \\
\hline \multirow[t]{4}{*}{ Pulau Weh } & $\mathrm{Na}$ & 5 & 6 & 2 & 3 & 6 & 5 & 2 & 1 & 1 \\
\hline & Но & 0.278 & 0.421 & 0.643 & 0.524 & 0.545 & 0.182 & 0.400 & 0.000 & 0.000 \\
\hline & $\mathrm{He}$ & 0.489 & 0.576 & 0.436 & 0.482 & 0.706 & 0.636 & 0.320 & 0.000 & 0.000 \\
\hline & $F_{\text {IS }}$ & 0.432 & 0.269 & -0.474 & -0.087 & 0.227 & 0.714 & -0.250 & - & - \\
\hline \multirow[t]{4}{*}{ Anak Krakatau } & $\mathrm{Na}$ & 7 & 6 & 3 & 2 & 8 & 4 & 6 & 4 & 5 \\
\hline & Ho & 0.647 & 0.286 & 0.429 & 0.118 & 0.556 & 0.176 & 0.563 & 0.455 & 0.143 \\
\hline & $\mathrm{He}$ & 0.740 & 0.696 & 0.401 & 0.484 & 0.756 & 0.265 & 0.711 & 0.640 & 0.370 \\
\hline & $F_{\text {IS }}$ & 0.126 & 0.590 & -0.070 & 0.757 & 0.265 & 0.333 & 0.209 & 0.290 & 0.614 \\
\hline \multirow[t]{4}{*}{ Anyer } & $\mathrm{Na}$ & 3 & 2 & 3 & 1 & 3 & 5 & 7 & 4 & 3 \\
\hline & Ho & 0.429 & 0.000 & 0.941 & 0.000 & 0.900 & 0.158 & 0.944 & 0.333 & 0.350 \\
\hline & $\mathrm{He}$ & 0.659 & 0.298 & 0.602 & 0.000 & 0.620 & 0.428 & 0.806 & 0.295 & 0.296 \\
\hline & $F_{\text {IS }}$ & 0.349 & 1.000 & -0.563 & - & -0.452 & 0.631 & -0.172 & -0.129 & -0.181 \\
\hline \multirow[t]{4}{*}{ Karang Serang } & $\mathrm{Na}$ & 5 & 4 & 2 & 2 & 7 & 5 & 4 & 6 & 6 \\
\hline & Ho & 0.563 & 0.467 & 0.667 & 0.118 & 0.529 & 0.471 & 0.462 & 0.300 & 0.083 \\
\hline & $\mathrm{He}$ & 0.764 & 0.429 & 0.444 & 0.484 & 0.740 & 0.578 & 0.689 & 0.795 & 0.684 \\
\hline & $F_{\text {IS }}$ & 0.263 & -0.088 & -0.500 & 0.757 & 0.285 & 0.186 & 0.330 & 0.623 & 0.878 \\
\hline \multirow[t]{4}{*}{ Rakata } & $\mathrm{Na}$ & 3 & 6 & 2 & 2 & 8 & 5 & 4 & 6 & 5 \\
\hline & Ho & 0.105 & 0.450 & 0.773 & 0.053 & 0.478 & 0.273 & 0.200 & 0.500 & 0.125 \\
\hline & $\mathrm{He}$ & 0.410 & 0.571 & 0.474 & 0.051 & 0.596 & 0.543 & 0.682 & 0.746 & 0.281 \\
\hline & $F_{\text {IS }}$ & 0.743 & 0.212 & -0.630 & -0.027 & 0.198 & 0.498 & 0.707 & 0.330 & 0.556 \\
\hline \multirow[t]{4}{*}{ Rakata Kecil } & $\mathrm{Na}$ & 5 & 6 & 2 & 5 & 10 & 5 & 5 & 6 & 5 \\
\hline & Ho & 0.842 & 0.350 & 0.105 & 0.800 & 0.952 & 0.150 & 0.538 & 0.438 & 0.353 \\
\hline & $\mathrm{He}$ & 0.620 & 0.628 & 0.100 & 0.680 & 0.745 & 0.349 & 0.544 & 0.785 & 0.545 \\
\hline & $F_{\text {IS }}$ & -0.357 & 0.442 & -0.056 & -0.176 & -0.279 & 0.570 & 0.011 & 0.443 & 0.352 \\
\hline \multirow[t]{4}{*}{ Sangiang } & $\mathrm{Na}$ & 6 & 4 & 3 & 4 & 6 & 4 & 7 & 6 & 5 \\
\hline & Ho & 0.375 & 0.158 & 0.250 & 0.400 & 0.800 & 0.136 & 0.391 & 0.810 & 0.208 \\
\hline & $\mathrm{He}$ & 0.689 & 0.506 & 0.223 & 0.490 & 0.742 & 0.685 & 0.590 & 0.680 & 0.359 \\
\hline & $F_{\text {IS }}$ & 0.456 & 0.688 & -0.121 & 0.183 & -0.079 & 0.801 & 0.337 & -0.190 & 0.419 \\
\hline \multirow[t]{4}{*}{ Sebuku 1} & $\mathrm{Na}$ & 3 & 6 & 2 & 2 & 6 & 3 & 6 & 5 & 1 \\
\hline & Ho & 0.400 & 0.550 & 0.400 & 0.350 & 0.579 & 0.211 & 0.529 & 0.750 & 0.000 \\
\hline & $\mathrm{He}$ & 0.591 & 0.729 & 0.320 & 0.439 & 0.769 & 0.436 & 0.690 & 0.734 & 0.000 \\
\hline & $F_{\text {IS }}$ & 0.323 & 0.245 & -0.250 & 0.202 & 0.247 & 0.517 & 0.233 & -0.021 & - \\
\hline \multirow[t]{4}{*}{ Sebuku 2} & $\mathrm{Na}$ & 3 & 5 & 2 & 2 & 8 & 4 & 7 & 0 & 2 \\
\hline & Ho & 0.571 & 0.409 & 0.529 & 0.476 & 0.696 & 0.476 & 0.526 & 0.000 & 0.063 \\
\hline & $\mathrm{He}$ & 0.625 & 0.616 & 0.389 & 0.490 & 0.836 & 0.670 & 0.687 & 0.000 & 0.061 \\
\hline & $F_{\text {IS }}$ & 0.086 & 0.336 & -0.360 & 0.028 & 0.168 & 0.289 & 0.234 & NA & -0.032 \\
\hline \multirow[t]{4}{*}{ Alam Kotok } & $\mathrm{Na}$ & 3 & 4 & 2 & 2 & 6 & 5 & 5 & 2 & 1 \\
\hline & Ho & 0.167 & 0.294 & 0.412 & 0.300 & 0.682 & 0.318 & 0.706 & 0.000 & 0.000 \\
\hline & $\mathrm{He}$ & 0.542 & 0.621 & 0.327 & 0.480 & 0.657 & 0.596 & 0.713 & 0.500 & 0.000 \\
\hline & $F_{\text {IS }}$ & 0.692 & 0.526 & -0.259 & 0.375 & -0.038 & 0.466 & 0.010 & 1.000 & - \\
\hline \multirow[t]{4}{*}{ Belat } & $\mathrm{Na}$ & 4 & 4 & 3 & 2 & 6 & 5 & 6 & 7 & 4 \\
\hline & Ho & 0.529 & 0.111 & 0.118 & 0.316 & 0.941 & 0.111 & 0.375 & 0.286 & 0.375 \\
\hline & $\mathrm{He}$ & 0.625 & 0.205 & 0.112 & 0.266 & 0.723 & 0.512 & 0.781 & 0.758 & 0.631 \\
\hline & $F_{\text {IS }}$ & 0.152 & 0.459 & -0.046 & -0.187 & -0.301 & 0.783 & 0.520 & 0.623 & 0.406 \\
\hline \multirow[t]{4}{*}{ Karang Congkak } & $\mathrm{Na}$ & 6 & 4 & 2 & 2 & 9 & 7 & 4 & 3 & 5 \\
\hline & Ho & 0.533 & 0.357 & 0.500 & 0.250 & 0.867 & 0.353 & 0.000 & 0.000 & 0.250 \\
\hline & $\mathrm{He}$ & 0.564 & 0.474 & 0.375 & 0.430 & 0.847 & 0.709 & 0.735 & 0.449 & 0.615 \\
\hline & $F_{\mathrm{IS}}$ & 0.055 & 0.247 & -0.333 & 0.418 & -0.024 & 0.502 & 1.000 & 1.000 & 0.594 \\
\hline
\end{tabular}


Table 2 continued

\begin{tabular}{|c|c|c|c|c|c|c|c|c|c|c|}
\hline & & $\mathrm{Pd} 2-001$ & $\mathrm{Pd} 3-002$ & $\mathrm{Pd} 2-003$ & Pd3-004 & $\mathrm{Pd} 3-005$ & $\mathrm{Pd} 2-006$ & Pv2 & Pv6 & Pv7 \\
\hline \multirow[t]{4}{*}{ Semak Daun } & $\mathrm{Na}$ & 4 & 5 & 2 & 4 & 11 & 6 & 4 & 6 & 3 \\
\hline & Ho & 0.526 & 0.647 & 0.200 & 0.500 & 0.857 & 0.263 & 0.476 & 0.438 & 0.111 \\
\hline & $\mathrm{He}$ & 0.654 & 0.663 & 0.255 & 0.583 & 0.746 & 0.593 & 0.638 & 0.689 & 0.204 \\
\hline & $F_{\text {IS }}$ & 0.195 & 0.023 & 0.216 & 0.143 & -0.149 & 0.556 & 0.254 & 0.365 & 0.455 \\
\hline \multirow[t]{4}{*}{ Seraya } & $\mathrm{Na}$ & 5 & 4 & 3 & 3 & 11 & 5 & 9 & 5 & 5 \\
\hline & Ho & 0.133 & 0.632 & 0.538 & 0.333 & 0.500 & 0.350 & 0.333 & 0.611 & 0.095 \\
\hline & $\mathrm{He}$ & 0.689 & 0.632 & 0.411 & 0.497 & 0.761 & 0.636 & 0.802 & 0.691 & 0.334 \\
\hline & $F_{\text {IS }}$ & 0.806 & 0.000 & -0.309 & 0.329 & 0.343 & 0.450 & 0.584 & 0.116 & 0.715 \\
\hline \multirow[t]{4}{*}{ Gili Trawangan } & $\mathrm{Na}$ & 4 & 4 & 4 & 2 & 5 & 3 & 10 & 6 & 3 \\
\hline & Ho & 0.444 & 0.556 & 0.765 & 0.471 & 0.417 & 0.000 & 0.722 & 0.500 & 0.091 \\
\hline & $\mathrm{He}$ & 0.406 & 0.691 & 0.528 & 0.360 & 0.694 & 0.639 & 0.634 & 0.727 & 0.244 \\
\hline & $F_{\text {IS }}$ & -0.095 & 0.196 & -0.449 & -0.308 & 0.400 & 1.000 & -0.139 & 0.312 & 0.627 \\
\hline \multirow[t]{4}{*}{ Barrang Lompo } & $\mathrm{Na}$ & 6 & 5 & 4 & 6 & 9 & 3 & 6 & 8 & 5 \\
\hline & Ho & 0.458 & 0.652 & 0.667 & 0.684 & 0.966 & 0.533 & 0.273 & 0.615 & 0.233 \\
\hline & $\mathrm{He}$ & 0.678 & 0.758 & 0.517 & 0.615 & 0.780 & 0.516 & 0.712 & 0.808 & 0.598 \\
\hline & $F_{\text {IS }}$ & 0.324 & 0.140 & -0.289 & -0.113 & -0.238 & -0.033 & 0.617 & 0.239 & 0.610 \\
\hline \multirow[t]{4}{*}{ Nudi Retreat } & $\mathrm{Na}$ & 4 & 4 & 2 & 4 & 8 & 6 & 7 & 6 & 2 \\
\hline & Ho & 0.174 & 0.640 & 0.520 & 0.200 & 0.640 & 0.240 & 0.542 & 0.684 & 0.269 \\
\hline & $\mathrm{He}$ & 0.518 & 0.556 & 0.385 & 0.284 & 0.677 & 0.713 & 0.765 & 0.729 & 0.233 \\
\hline & $F_{\mathrm{IS}}$ & 0.664 & -0.151 & -0.351 & 0.296 & 0.054 & 0.663 & 0.292 & 0.061 & -0.156 \\
\hline \multirow[t]{4}{*}{ Jerewai } & $\mathrm{Na}$ & 4 & 6 & 4 & 3 & 7 & 4 & 4 & 5 & 4 \\
\hline & Ho & 0.533 & 0.438 & 0.438 & 0.143 & 0.467 & 0.500 & 0.500 & 0.182 & 0.222 \\
\hline & $\mathrm{He}$ & 0.629 & 0.578 & 0.363 & 0.255 & 0.533 & 0.619 & 0.695 & 0.740 & 0.617 \\
\hline & $F_{\mathrm{IS}}$ & 0.152 & 0.243 & -0.204 & 0.440 & 0.125 & 0.192 & 0.281 & 0.754 & 0.640 \\
\hline \multirow[t]{4}{*}{ Tonuu } & $\mathrm{Na}$ & 3 & 6 & 2 & 4 & 8 & 3 & 5 & 4 & 4 \\
\hline & Ho & 0.143 & 0.842 & 0.222 & 0.700 & 0.850 & 0.250 & 0.231 & 0.250 & 0.273 \\
\hline & $\mathrm{He}$ & 0.253 & 0.665 & 0.198 & 0.536 & 0.785 & 0.656 & 0.642 & 0.719 & 0.616 \\
\hline & $F_{\mathrm{IS}}$ & 0.434 & -0.267 & -0.125 & -0.305 & -0.083 & 0.619 & 0.641 & 0.652 & 0.557 \\
\hline \multirow[t]{4}{*}{ Alyui } & $\mathrm{Na}$ & 2 & 4 & 2 & 3 & 5 & 2 & 3 & 4 & 2 \\
\hline & Ho & 0.200 & 0.947 & 0.105 & 0.944 & 0.842 & 0.158 & 1.000 & 1.000 & 0.111 \\
\hline & $\mathrm{He}$ & 0.180 & 0.569 & 0.100 & 0.523 & 0.727 & 0.145 & 0.569 & 0.652 & 0.105 \\
\hline & $F_{\mathrm{IS}}$ & -0.111 & -0.664 & -0.056 & -0.805 & -0.158 & -0.086 & -0.756 & -0.533 & -0.059 \\
\hline \multirow[t]{4}{*}{ Jefman } & $\mathrm{Na}$ & 5 & 4 & 4 & 5 & 8 & 6 & 5 & 8 & 5 \\
\hline & Ho & 0.467 & 0.182 & 0.500 & 0.318 & 0.667 & 0.250 & 0.556 & 0.474 & 0.238 \\
\hline & $\mathrm{He}$ & 0.698 & 0.591 & 0.409 & 0.527 & 0.709 & 0.500 & 0.715 & 0.823 & 0.747 \\
\hline & $F_{\mathrm{IS}}$ & 0.331 & 0.692 & -0.223 & 0.396 & 0.059 & 0.500 & 0.222 & 0.424 & 0.681 \\
\hline \multirow[t]{4}{*}{ Kri } & $\mathrm{Na}$ & 3 & 7 & 3 & 6 & 10 & 4 & 9 & 8 & 2 \\
\hline & Ho & 0.227 & 0.655 & 0.571 & 0.690 & 0.833 & 0.462 & 0.654 & 0.538 & 0.036 \\
\hline & $\mathrm{He}$ & 0.334 & 0.691 & 0.426 & 0.566 & 0.834 & 0.675 & 0.805 & 0.814 & 0.270 \\
\hline & $F_{\mathrm{IS}}$ & 0.319 & 0.052 & -0.341 & -0.218 & 0.001 & 0.316 & 0.188 & 0.338 & 0.868 \\
\hline \multirow[t]{4}{*}{ Ambai } & $\mathrm{Na}$ & 3 & 4 & 3 & 5 & 8 & 3 & 4 & 7 & 5 \\
\hline & Ho & 0.214 & 0.308 & 0.267 & 0.357 & 0.867 & 0.375 & 0.273 & 0.357 & 0.143 \\
\hline & $\mathrm{He}$ & 0.548 & 0.559 & 0.238 & 0.651 & 0.742 & 0.320 & 0.657 & 0.786 & 0.768 \\
\hline & $F_{\text {IS }}$ & 0.609 & 0.450 & -0.121 & 0.451 & -0.168 & -0.171 & 0.585 & 0.545 & 0.814 \\
\hline \multirow[t]{4}{*}{ Lemon } & $\mathrm{Na}$ & 6 & 2 & 3 & 2 & 5 & 3 & 3 & 7 & 7 \\
\hline & Но & 0.235 & 0.091 & 0.800 & 0.625 & 0.167 & 0.059 & 0.941 & 0.889 & 0.250 \\
\hline & $\mathrm{He}$ & 0.360 & 0.087 & 0.504 & 0.430 & 0.340 & 0.299 & 0.628 & 0.718 & 0.508 \\
\hline & $F_{\text {IS }}$ & 0.346 & -0.048 & -0.586 & -0.455 & 0.509 & 0.803 & -0.499 & -0.239 & 0.508 \\
\hline
\end{tabular}


Table 2 continued

\begin{tabular}{llllllllllll}
\hline & & Pd2-001 & Pd3-002 & Pd2-003 & Pd3-004 & Pd3-005 & Pd2-006 & Pv2 & Pv6 & Pv7 \\
\hline Mauwara & $\mathrm{Na}$ & 7 & 4 & 4 & 3 & 5 & 4 & 7 & 6 & 6 \\
& $\mathrm{Ho}$ & 0.522 & 0.278 & 0.476 & 0.409 & 0.706 & 0.071 & 0.909 & 0.429 & 0.316 \\
& $\mathrm{He}$ & 0.629 & 0.514 & 0.500 & 0.334 & 0.715 & 0.671 & 0.767 & 0.731 & 0.500 \\
& $F_{\text {IS }}$ & 0.170 & 0.459 & 0.048 & -0.226 & 0.012 & 0.894 & -0.186 & 0.414 & 0.368 \\
\hline
\end{tabular}

Shown for each locus and locality are the number of alleles observed (A), observed heterozygosity (Ho), and expected heterozygosity (He). $F_{\text {IS }}$ is the inbreeding coefficient. Values in italics indicate significant departures from Hardy-Weinberg equilibrium after FDR correction

disequilibrium was detected in some cases, but was not consistent across loci and study localities and no two loci were linked in all localities (data not shown). Underwood et al. (2007) and van Oppen et al. (2008) attribute inconsistent Hardy-Weinberg and linkage disequilibrium in S. hystrix from Western Australia and the Great Barrier Reef to genetic subdivision at the local scale, combined with admixture of populations, and concluded that null alleles were not a cause of the heterozygote deficits. High levels of genetic subdivision at the local scale in both $P$. damicornis and $S$. hystrix in Krakatau and throughout Indonesia (see results from BAPS 5.3) lead to the same conclusion here and therefore did not preclude further analyses with these data.

The number of unique genotypes observed, Nei's unbiased gene diversity (Hz), $M$ value, and BAPS cluster for each population are given in Tables 4 and 5. Nearly all localities in Krakatau display $M$ statistics below 7 indicating recent reductions in population size (Garza and Williamson 2001). Average $M$ in localities across Indonesia was 0.67 for $P$. damicornis (Table 4) and 0.83 for S. hystrix (Table 5).

When comparing the two species to each other at the 4 localities within Krakatau where both species were sampled, Nei's unbiased gene diversity ( $\mathrm{Hz}$ was greater for $P$. damicornis (Table 4) than in S. hystrix (Table 5) in all comparisons (Wilcoxon signed rank test, $p=0.05$ ). When comparing localities across all of Indonesia where both species were sampled, gene diversity is also significantly higher in $P$. damicornis (mean $\mathrm{Hz}=0.57$ ) than in $S$. hystrix (mean $\mathrm{Hz}=0.45$ ) (Wilcoxon signed rank test, $p=0.00)$.

When comparing localities within Krakatau to the remaining localities throughout Indonesia for $P$. damicornis, genetic diversity localities in Krakatau (mean $\mathrm{Hz}=0.55$ ) was marginally higher than the mean $\mathrm{Hz}$ for the remaining localities throughout Indonesia (mean $\mathrm{Hz}=0.53$ ) (Table 4). The mean genetic diversity of $S$. hystrix localities in Krakatau (mean $\mathrm{Hz}=0.41$ ) was lower than the mean of the remaining localities throughout Indonesia (mean $\mathrm{Hz}=0.48$ ) (Table 5).
Assignment methods

For both species, there was strong agreement between the spatial and non-spatial assignment methods implemented in BAPS. Likelihood was higher in the non-spatial analysis for $P$. damicornis $(-11,587.93$ vs. $-11,743.45)$ and S. hystrix (-11,098.75 vs. $-11,345.53)$ indicating that the molecular data alone were adequate to resolve genetic structure and that departures from HWE did not spuriously affect the results. Each locality was assigned to a genetic cluster, given in Fig. 2a, b and Tables 4 and 5. The conditional posterior probabilities of assignment were all greater than or equal to 0.99 indicating the highest probability of membership.

The Bayesian assignment method implemented in BAPS indicated the presence of $K=16$ genetic clusters from 26 localities of $P$. damicornis in Indonesia (Table 4), 5 of which occur in Krakatau. Three of these clusters are found in one locality each and are private to the Sunda Strait: Rakata, Sangiang, and Anyer. Three localities in Krakatau (Krakata Kecil, Sebuku 1 and Sebuku 2) cluster with 2 localities in Pulau Seribu (Alam Kotok and Semak Daun) to form Cluster \#1 (Table 4). Anak Krakatau and Karang Serang together form Cluster \#6, which is found nowhere else in Indonesia.

The Bayesian assignment method implemented in BAPS indicated the presence of $K=20$ genetic clusters from 31 localities of $S$. hystrix in Indonesia (Table 5), only one of which occurs in Krakatau. S. hystrix from Sangiang, Anak Krakatau, Sebuku 1, Sebuku 2 form Cluster \#2 with all localities from Pulau Seribu: Alam Kotok, Pramuka, Semak Daun, Karang Kongka, Belat, Pulau Pari, This cluster occurs nowhere else in Indonesia.

Genetic structure and migration

In $P$. damicornis, pairwise $F_{\text {st }}$ was significant between Krakatau and 11 out of 13 other regions (Table 6). The smallest pairwise $F_{\text {st }}$ values were between Krakatau and Pulau Seribu $\left(F_{\text {st }}=0.03\right)$ and Krakatau and Bali $\left(F_{\text {st }}=0.03\right)$. In $S$. hystrix, pairwise $F_{\text {st }}$ estimates were 
Table 3 Allele data for $S$. hystrix for populations where $\mathrm{Ng} \geq 15$

\begin{tabular}{|c|c|c|c|c|c|c|c|c|}
\hline & & Sh2-005 & Sh2-006 & Sh3-003 & Sh3-004 & Sh3-008 & Sh4-001 & Sh4-010 \\
\hline \multirow[t]{4}{*}{ Pulau Weh } & $\mathrm{Na}$ & 5 & 3 & 2 & 3 & 2 & 2 & 2 \\
\hline & Ho & 0.720 & 0.320 & 0.000 & 0.360 & 0.000 & 0.200 & 0.467 \\
\hline & $\mathrm{He}$ & 0.608 & 0.594 & 0.493 & 0.627 & 0.497 & 0.241 & 0.420 \\
\hline & $F_{\text {IS }}$ & -0.184 & 0.462 & 1.000 & 0.426 & 1.000 & 0.169 & -0.111 \\
\hline \multirow[t]{4}{*}{ Anak Krakatau } & $\mathrm{Na}$ & 4 & 6 & 1 & 6 & 1 & 5 & 5 \\
\hline & Ho & 0.682 & 0.636 & 0.000 & 0.476 & 0.000 & 0.238 & 0.500 \\
\hline & $\mathrm{He}$ & 0.561 & 0.667 & 0.000 & 0.520 & 0.000 & 0.298 & 0.477 \\
\hline & $F_{\text {IS }}$ & -0.215 & 0.046 & - & 0.085 & - & 0.202 & -0.048 \\
\hline \multirow[t]{4}{*}{ Rakata } & $\mathrm{Na}$ & 3 & 4 & 1 & 4 & 1 & 2 & 5 \\
\hline & Ho & 1.000 & 0.167 & 0.000 & 0.556 & 0.000 & 0.389 & 0.611 \\
\hline & $\mathrm{He}$ & 0.549 & 0.157 & 0.000 & 0.545 & 0.000 & 0.375 & 0.600 \\
\hline & $F_{\text {IS }}$ & -0.820 & -0.059 & - & -0.020 & - & -0.037 & -0.018 \\
\hline \multirow[t]{4}{*}{ Sangiang } & $\mathrm{Na}$ & 5 & 6 & 1 & 4 & 2 & 3 & 4 \\
\hline & Ho & 0.708 & 0.542 & 0.000 & 0.696 & 0.000 & 0.261 & 0.542 \\
\hline & $\mathrm{He}$ & 0.734 & 0.752 & 0.000 & 0.665 & 0.080 & 0.235 & 0.489 \\
\hline & $F_{\text {IS }}$ & 0.034 & 0.279 & - & -0.045 & 1.000 & -0.108 & -0.108 \\
\hline \multirow[t]{4}{*}{ Sebuku 1} & $\mathrm{Na}$ & 6 & 5 & 3 & 7 & 1 & 4 & 6 \\
\hline & Ho & 0.870 & 0.583 & 0.000 & 0.478 & 0.000 & 0.238 & 0.458 \\
\hline & $\mathrm{He}$ & 0.698 & 0.497 & 0.156 & 0.682 & 0.000 & 0.330 & 0.635 \\
\hline & $F_{\text {IS }}$ & -0.245 & -0.175 & 1.000 & 0.299 & - & 0.278 & 0.278 \\
\hline \multirow[t]{4}{*}{ Sebuku 2} & $\mathrm{Na}$ & 7 & 5 & 2 & 2 & 2 & 2 & 4 \\
\hline & Ho & 0.957 & 0.560 & 0.000 & 0.476 & 0.080 & 0.727 & 0.625 \\
\hline & $\mathrm{He}$ & 0.730 & 0.567 & 0.077 & 0.444 & 0.077 & 0.463 & 0.470 \\
\hline & $F_{\text {IS }}$ & -0.311 & 0.013 & 1.000 & -0.071 & -0.042 & -0.571 & -0.328 \\
\hline \multirow[t]{4}{*}{ Alam Kotok } & $\mathrm{Na}$ & 7 & 4 & 2 & 4 & 2 & 2 & 4 \\
\hline & Ho & 0.850 & 0.450 & 0.050 & 0.650 & 0.000 & 0.368 & 0.400 \\
\hline & $\mathrm{He}$ & 0.694 & 0.558 & 0.049 & 0.548 & 0.198 & 0.301 & 0.475 \\
\hline & $F_{\text {IS }}$ & -0.225 & 0.193 & -0.026 & -0.187 & 1.000 & -0.226 & 0.158 \\
\hline \multirow[t]{4}{*}{ Belat } & $\mathrm{Na}$ & 6 & 6 & 1 & 2 & 3 & 3 & 7 \\
\hline & Ho & 0.842 & 0.684 & 0.000 & 0.524 & 0.053 & 0.100 & 0.526 \\
\hline & $\mathrm{He}$ & 0.755 & 0.668 & 0.000 & 0.482 & 0.483 & 0.096 & 0.634 \\
\hline & $F_{\text {IS }}$ & -0.116 & -0.025 & - & -0.087 & 0.891 & -0.039 & 0.170 \\
\hline \multirow[t]{4}{*}{ Karang Congkak } & $\mathrm{Na}$ & 7 & 5 & 1 & 5 & 1 & 2 & 6 \\
\hline & Ho & 1.000 & 0.667 & 0.000 & 0.714 & 0.000 & 0.048 & 0.571 \\
\hline & $\mathrm{He}$ & 0.752 & 0.745 & 0.000 & 0.622 & 0.000 & 0.046 & 0.635 \\
\hline & $F_{\text {IS }}$ & -0.330 & 0.105 & - & -0.148 & - & -0.024 & 0.100 \\
\hline \multirow[t]{4}{*}{ Pramuka } & $\mathrm{Na}$ & 6 & 7 & 1 & 3 & 1 & 3 & 4 \\
\hline & Ho & 0.913 & 0.739 & 0.000 & 0.391 & 0.000 & 0.348 & 0.609 \\
\hline & $\mathrm{He}$ & 0.665 & 0.582 & 0.000 & 0.519 & 0.000 & 0.360 & 0.691 \\
\hline & $F_{\text {IS }}$ & -0.372 & -0.269 & - & 0.246 & - & 0.034 & 0.119 \\
\hline \multirow[t]{4}{*}{ Pulau Pari } & $\mathrm{Na}$ & 7 & 6 & 3 & 8 & 3 & 3 & 6 \\
\hline & Ho & 0.692 & 0.667 & 0.024 & 0.711 & 0.024 & 0.514 & 0.550 \\
\hline & $\mathrm{He}$ & 0.795 & 0.713 & 0.156 & 0.817 & 0.116 & 0.480 & 0.614 \\
\hline & $F_{\text {IS }}$ & 0.129 & 0.065 & 0.848 & 0.131 & 0.789 & -0.069 & 0.104 \\
\hline \multirow[t]{4}{*}{ Semak Daun } & $\mathrm{Na}$ & 7 & 6 & 1 & 5 & 3 & 4 & 4 \\
\hline & Ho & 1.000 & 0.667 & 0.000 & 0.810 & 0.048 & 0.350 & 0.619 \\
\hline & $\mathrm{He}$ & 0.766 & 0.681 & 0.000 & 0.668 & 0.441 & 0.303 & 0.586 \\
\hline & $F_{\text {IS }}$ & -0.305 & 0.022 & - & -0.212 & 0.892 & -0.157 & -0.056 \\
\hline
\end{tabular}


Table 3 continued

\begin{tabular}{|c|c|c|c|c|c|c|c|c|}
\hline & & Sh2-005 & Sh2-006 & Sh3-003 & Sh3-004 & Sh3-008 & Sh4-001 & Sh4-010 \\
\hline \multirow[t]{4}{*}{ Napoleon Reef } & $\mathrm{Na}$ & 6 & 7 & 1 & 6 & 2 & 2 & 5 \\
\hline & Но & 0.900 & 0.722 & 0.000 & 0.524 & 0.050 & 0.476 & 0.632 \\
\hline & $\mathrm{He}$ & 0.785 & 0.728 & 0.000 & 0.624 & 0.049 & 0.444 & 0.507 \\
\hline & $F_{\text {IS }}$ & -0.146 & 0.008 & - & 0.160 & -0.026 & -0.071 & -0.246 \\
\hline \multirow[t]{4}{*}{ Pemuteran } & $\mathrm{Na}$ & 6 & 4 & 1 & 4 & 3 & 4 & 5 \\
\hline & Ho & 0.810 & 0.575 & 0.000 & 0.564 & 0.026 & 0.314 & 0.711 \\
\hline & $\mathrm{He}$ & 0.622 & 0.514 & 0.000 & 0.645 & 0.075 & 0.278 & 0.678 \\
\hline & $F_{\text {IS }}$ & -0.301 & -0.118 & - & 0.126 & 0.656 & -0.132 & -0.049 \\
\hline \multirow[t]{4}{*}{ Seraya } & $\mathrm{Na}$ & 6 & 5 & 1 & 4 & 2 & 3 & 4 \\
\hline & Ho & 0.450 & 0.526 & 0.000 & 0.588 & 0.105 & 0.364 & 0.800 \\
\hline & $\mathrm{He}$ & 0.488 & 0.472 & 0.000 & 0.618 & 0.332 & 0.310 & 0.603 \\
\hline & $F_{\text {IS }}$ & 0.077 & -0.114 & - & 0.048 & 0.683 & -0.173 & -0.328 \\
\hline \multirow[t]{4}{*}{ Bone Batang } & $\mathrm{Na}$ & 5 & 6 & 2 & 9 & 4 & 5 & 3 \\
\hline & Ho & 0.636 & 0.682 & 0.000 & 0.750 & 0.095 & 0.167 & 0.174 \\
\hline & $\mathrm{He}$ & 0.718 & 0.769 & 0.100 & 0.853 & 0.531 & 0.622 & 0.162 \\
\hline & $F_{\text {IS }}$ & 0.114 & 0.113 & 1.000 & 0.120 & 0.821 & 0.732 & -0.076 \\
\hline \multirow[t]{4}{*}{ Selayar } & $\mathrm{Na}$ & 6 & 4 & 1 & 4 & 2 & 4 & 3 \\
\hline & Ho & 0.500 & 0.267 & 0.000 & 0.778 & 0.333 & 0.063 & 0.733 \\
\hline & $\mathrm{He}$ & 0.762 & 0.691 & 0.000 & 0.616 & 0.491 & 0.561 & 0.540 \\
\hline & $F_{\mathrm{IS}}$ & 0.344 & 0.614 & - & -0.263 & 0.321 & 0.889 & -0.358 \\
\hline \multirow[t]{4}{*}{ Sebayur } & $\mathrm{Na}$ & 9 & 5 & 1 & 6 & 4 & 3 & 7 \\
\hline & Ho & 0.938 & 0.500 & 0.000 & 0.588 & 0.308 & 0.533 & 0.529 \\
\hline & $\mathrm{He}$ & 0.760 & 0.635 & 0.000 & 0.737 & 0.648 & 0.531 & 0.554 \\
\hline & $F_{\text {IS }}$ & -0.234 & 0.213 & - & 0.202 & 0.525 & -0.004 & 0.044 \\
\hline \multirow[t]{4}{*}{ Bangka (Murex) } & $\mathrm{Na}$ & 8 & 6 & 6 & 9 & 3 & 4 & 7 \\
\hline & Но & 0.676 & 0.382 & 0.259 & 0.682 & 0.000 & 0.360 & 0.667 \\
\hline & $\mathrm{He}$ & 0.812 & 0.760 & 0.460 & 0.819 & 0.362 & 0.682 & 0.786 \\
\hline & $F_{\text {IS }}$ & 0.167 & 0.497 & 0.436 & 0.168 & 1.000 & 0.472 & 0.152 \\
\hline \multirow[t]{4}{*}{ Nudi Retreat } & $\mathrm{Na}$ & 10 & 8 & 3 & 8 & 3 & 9 & 9 \\
\hline & Но & 0.829 & 0.686 & 0.229 & 0.829 & 0.094 & 0.529 & 0.697 \\
\hline & $\mathrm{He}$ & 0.822 & 0.836 & 0.295 & 0.820 & 0.090 & 0.749 & 0.803 \\
\hline & $F_{\text {IS }}$ & -0.007 & 0.180 & 0.225 & -0.010 & -0.038 & 0.293 & 0.132 \\
\hline \multirow[t]{4}{*}{ Doi } & $\mathrm{Na}$ & 11 & 6 & 3 & 7 & 3 & 4 & 3 \\
\hline & Ho & 0.550 & 0.737 & 0.100 & 0.632 & 0.150 & 0.368 & 0.300 \\
\hline & $\mathrm{He}$ & 0.711 & 0.778 & 0.184 & 0.809 & 0.141 & 0.464 & 0.516 \\
\hline & $F_{\mathrm{IS}}$ & 0.227 & 0.053 & 0.456 & 0.219 & -0.062 & 0.206 & 0.419 \\
\hline \multirow[t]{4}{*}{ Jerewai } & $\mathrm{Na}$ & 6 & 3 & 2 & 5 & 1 & 5 & 5 \\
\hline & Ho & 0.636 & 0.400 & 0.227 & 0.409 & 0.000 & 0.182 & 0.864 \\
\hline & $\mathrm{He}$ & 0.614 & 0.580 & 0.201 & 0.594 & 0.000 & 0.319 & 0.670 \\
\hline & $F_{\text {IS }}$ & -0.037 & 0.310 & -0.128 & 0.311 & - & 0.430 & -0.288 \\
\hline \multirow[t]{4}{*}{ Tidore } & $\mathrm{Na}$ & 4 & 4 & 1 & 4 & 1 & 4 & 3 \\
\hline & Ho & 0.107 & 1.000 & 0.000 & 0.444 & 0.000 & 0.143 & 0.571 \\
\hline & $\mathrm{He}$ & 0.136 & 0.549 & 0.000 & 0.568 & 0.000 & 0.136 & 0.433 \\
\hline & $F_{\text {IS }}$ & 0.211 & -0.821 & - & 0.217 & - & -0.052 & -0.320 \\
\hline \multirow[t]{4}{*}{ Tonuu } & $\mathrm{Na}$ & 6 & 5 & 2 & 8 & 2 & 2 & 3 \\
\hline & Но & 0.526 & 0.632 & 0.050 & 0.947 & 0.526 & 0.158 & 0.526 \\
\hline & $\mathrm{He}$ & 0.565 & 0.547 & 0.049 & 0.780 & 0.465 & 0.145 & 0.497 \\
\hline & $F_{\text {IS }}$ & 0.069 & -0.154 & -0.026 & -0.215 & -0.131 & -0.086 & -0.058 \\
\hline
\end{tabular}


Table 3 continued

\begin{tabular}{|c|c|c|c|c|c|c|c|c|}
\hline & & Sh2-005 & Sh2-006 & Sh3-003 & Sh3-004 & Sh3-008 & Sh4-001 & Sh4-010 \\
\hline \multirow[t]{4}{*}{ Alyui } & $\mathrm{Na}$ & 6 & 7 & 1 & 5 & 3 & 4 & 3 \\
\hline & Но & 0.588 & 0.375 & 0.000 & 0.684 & 0.167 & 0.474 & 0.632 \\
\hline & $\mathrm{He}$ & 0.685 & 0.791 & 0.000 & 0.532 & 0.403 & 0.537 & 0.644 \\
\hline & $F_{\mathrm{IS}}$ & 0.141 & 0.526 & - & -0.286 & 0.586 & 0.119 & 0.019 \\
\hline \multirow[t]{4}{*}{ Jefman } & $\mathrm{Na}$ & 7 & 5 & 1 & 4 & 1 & 4 & 5 \\
\hline & Ho & 1.000 & 0.364 & 0.000 & 0.727 & 0.000 & 0.190 & 0.773 \\
\hline & $\mathrm{He}$ & 0.716 & 0.543 & 0.000 & 0.691 & 0.000 & 0.178 & 0.675 \\
\hline & $F_{\text {IS }}$ & -0.397 & 0.331 & - & -0.052 & - & -0.070 & -0.145 \\
\hline \multirow[t]{4}{*}{ Mayalibit } & $\mathrm{Na}$ & 4 & 8 & 1 & 2 & 3 & 2 & 2 \\
\hline & Ho & 0.650 & 0.375 & 0.000 & 0.400 & 0.278 & 0.500 & 0.400 \\
\hline & $\mathrm{He}$ & 0.499 & 0.729 & 0.000 & 0.320 & 0.356 & 0.375 & 0.320 \\
\hline & $F_{\text {IS }}$ & -0.303 & 0.485 & - & -0.250 & 0.221 & -0.333 & -0.250 \\
\hline \multirow[t]{4}{*}{ Ambai } & $\mathrm{Na}$ & 8 & 6 & 2 & 6 & 3 & 3 & 2 \\
\hline & Ho & 0.773 & 0.286 & 0.045 & 0.545 & 0.182 & 0.214 & 0.045 \\
\hline & $\mathrm{He}$ & 0.798 & 0.707 & 0.044 & 0.694 & 0.549 & 0.253 & 0.044 \\
\hline & $F_{\text {IS }}$ & 0.031 & 0.596 & -0.023 & 0.214 & 0.669 & 0.152 & -0.023 \\
\hline \multirow[t]{4}{*}{ Lemon } & $\mathrm{Na}$ & 10 & 10 & 1 & 4 & 3 & 2 & 5 \\
\hline & Ho & 0.900 & 0.500 & 0.000 & 0.550 & 0.105 & 0.350 & 0.350 \\
\hline & $\mathrm{He}$ & 0.788 & 0.838 & 0.000 & 0.571 & 0.400 & 0.489 & 0.348 \\
\hline & $F_{\mathrm{IS}}$ & -0.143 & 0.403 & - & 0.037 & 0.737 & 0.284 & -0.007 \\
\hline \multirow[t]{4}{*}{ Rumberpon } & $\mathrm{Na}$ & 5 & 2 & 1 & 4 & 4 & 3 & 3 \\
\hline & Ho & 0.706 & 0.500 & 0.000 & 0.417 & 0.278 & 0.063 & 0.250 \\
\hline & $\mathrm{He}$ & 0.739 & 0.375 & 0.000 & 0.462 & 0.622 & 0.432 & 0.227 \\
\hline & $F_{\mathrm{IS}}$ & 0.044 & -0.333 & - & 0.098 & 0.553 & 0.855 & -0.103 \\
\hline \multirow[t]{4}{*}{ Namatote } & $\mathrm{Na}$ & 8 & 7 & 1 & 3 & 3 & 2 & 3 \\
\hline & Ho & 0.762 & 0.650 & 0.000 & 0.524 & 0.050 & 0.235 & 0.381 \\
\hline & $\mathrm{He}$ & 0.785 & 0.711 & 0.000 & 0.475 & 0.141 & 0.291 & 0.390 \\
\hline & $F_{\text {IS }}$ & 0.029 & 0.086 & - & -0.103 & 0.646 & 0.190 & 0.023 \\
\hline \multirow[t]{4}{*}{ Mommon } & $\mathrm{Na}$ & 7 & 6 & 1 & 5 & 3 & 4 & 3 \\
\hline & Но & 0.500 & 0.533 & 0.000 & 0.500 & 0.333 & 0.267 & 0.500 \\
\hline & $\mathrm{He}$ & 0.653 & 0.596 & 0.000 & 0.750 & 0.549 & 0.673 & 0.565 \\
\hline & $F_{\mathrm{IS}}$ & 0.234 & 0.104 & - & 0.333 & 0.393 & 0.604 & 0.115 \\
\hline
\end{tabular}

Shown for each locus and locality are the number of alleles observed (A), observed heterozygosity (Ho), and expected heterozygosity (He). $F_{\text {IS }}$ is the inbreeding coefficient. Values in italics indicate significant departures from Hardy-Weinberg equilibrium after FDR correction

significant between Krakatau and all other regions (Table 7). The lowest pairwise $F_{\text {st }}$ values were observed between Krakatau and Pulau Seribu $\left(F_{\text {st }}=0.02\right)$. The second lowest $F_{\text {st }}$ value was between Krakatau and Bali $\left(F_{\mathrm{st}}=0.04\right)$.

Immigration rates inferred from BayesAss + (Tables 8 and 9) indicate that $P$. damicornis populations in Krakatau are $96 \%$ self-seeding with the remaining larval input from distant sources. In addition, Krakatau is identified as a larval source for P. damicornis populations in Aceh, Pulau Seribu, and Lombok. S. hystrix in Krakatau is $75 \%$ selfseeding with $23 \%$ of its larvae immigrating from Pulau Seribu. Krakatau is identified as a possible larval source for S. hystrix populations in Biak.

\section{Discussion}

Comparing levels of genetic diversity between Krakatau and other regions

Following the complete destruction of Krakatau in 1883 and the extermination of all marine life on the surrounding islands, genetic diversity has largely recovered for two species of reef-building corals through larval migration from the nearby upstream reefs of Pulau Seribu, and to a lesser extent from Bali and more distant sites in Indonesia. However, many populations in Krakatau do fall below the mean diversity values for Indonesia indicating either that recovery is not complete, or that diversity has declined 
Table 4 P. damicornis locality statistics

\begin{tabular}{|c|c|c|c|c|c|c|c|}
\hline Locality & $\mathrm{N}$ & $\mathrm{Ng}$ & $\mathrm{Hz}$ & $\mathrm{Hz} \mathrm{SD}$ & $M$ & $M \mathrm{SD}$ & BAPS cluster \\
\hline Pulau Weh & 22 & 22 & 0.42 & 0.09 & 0.81 & 0.27 & 15 \\
\hline Anak Krakatau & 19 & 19 & 0.58 & 0.06 & 0.74 & 0.31 & 6 \\
\hline Anyer & 21 & 20 & 0.46 & 0.08 & 0.69 & 0.28 & 9 \\
\hline Karang Serang & 17 & 17 & 0.65 & 0.05 & 0.66 & 0.27 & 6 \\
\hline Rakata (Krakatau Besar) & 26 & 26 & 0.50 & 0.07 & 0.68 & 0.29 & 2 \\
\hline Rakata Kecil & 24 & 24 & 0.57 & 0.07 & 0.67 & 0.28 & 1 \\
\hline Sangiang & 25 & 24 & 0.56 & 0.06 & 0.62 & 0.22 & 10 \\
\hline Sebesi & 11 & 11 & NA & NA & NA & NA & NA \\
\hline Sebuku 1 & 20 & 20 & 0.54 & 0.09 & 0.67 & 0.30 & 1 \\
\hline Sebuku 2 & 23 & 23 & 0.56 & 0.09 & 0.62 & 0.35 & 1 \\
\hline Alam Kotok & 22 & 22 & 0.52 & 0.08 & 0.75 & 0.28 & 1 \\
\hline Belat & 20 & 20 & 0.53 & 0.09 & 0.68 & 0.26 & 11 \\
\hline Karang Congkak & 18 & 18 & 0.60 & 0.06 & 0.74 & 0.28 & 11 \\
\hline Pramuka & 10 & 10 & NA & NA & NA & NA & NA \\
\hline Pulau Pari & 13 & 13 & NA & NA & NA & NA & NA \\
\hline Semak Daun & 23 & 23 & 0.57 & 0.07 & 0.65 & 0.23 & 1 \\
\hline Seraya & 21 & 21 & 0.62 & 0.05 & 0.71 & 0.26 & 13 \\
\hline Pemuteran & 11 & 11 & 0.06 & 1.94 & 0.67 & 0.2 & NA \\
\hline Gili Trawangan & 18 & 18 & 0.57 & 0.06 & 0.71 & 0.24 & 14 \\
\hline Barrang Lompo & 30 & 30 & 0.68 & 0.04 & 0.75 & 0.23 & 12 \\
\hline Murex House Reef (Bangka) & 2 & 2 & NA & NA & NA & NA & NA \\
\hline Nudi Retreat & 26 & 26 & 0.55 & 0.07 & 0.60 & 0.24 & 8 \\
\hline Tanjung Pisok & 25 & 21 & 0.50 & 0.07 & 0.71 & 0.29 & 8 \\
\hline Manado Tua & 2 & 2 & NA & NA & NA & NA & NA \\
\hline Jerewai & 17 & 17 & 0.58 & 0.05 & 0.71 & 0.26 & 3 \\
\hline Tidore & 11 & 11 & NA & NA & NA & NA & NA \\
\hline Tonuu & 21 & 21 & 0.60 & 0.07 & 0.64 & 0.28 & 7 \\
\hline Alyui & 20 & 16 & 0.41 & 0.09 & 0.61 & 0.27 & 16 \\
\hline Gam & 4 & 4 & NA & NA & NA & NA & NA \\
\hline Jefman & 22 & 22 & 0.65 & 0.05 & 0.72 & 0.25 & 3 \\
\hline Kri & 30 & 30 & 0.60 & 0.09 & 0.68 & 0.30 & 7 \\
\hline Manta Point & 1 & 1 & NA & NA & NA & NA & NA \\
\hline Mayalibit & 9 & 9 & NA & NA & NA & NA & NA \\
\hline Adoki & 10 & 8 & NA & NA & NA & NA & NA \\
\hline Owi & 9 & 9 & NA & NA & NA & NA & NA \\
\hline Ambai & 15 & 15 & 0.61 & 0.07 & 0.62 & 0.22 & 3 \\
\hline Serui & 6 & 6 & NA & NA & NA & NA & NA \\
\hline Lemon & 18 & 18 & 0.44 & 0.06 & 0.64 & 0.28 & 4 \\
\hline Rumberpon & 13 & 13 & NA & NA & NA & NA & NA \\
\hline Mauwara & 23 & 21 & 0.61 & 0.05 & 0.59 & 0.23 & 5 \\
\hline Mommon & 4 & 4 & NA & NA & NA & NA & NA \\
\hline
\end{tabular}

Shown for each locality are the number of samples taken $(\mathrm{N})$, the number of unique genotypes observed $(\mathrm{Ng})$, Nei's unbiased gene diversity $(\mathrm{Hz})$ and standard deviation, Garza-Williams statistic value $(M)$ and standard deviation, and the cluster to which each population was assigned by BAPS. Conditional posterior probabilities of assignments were all $\geq 0.99$. Results are presented for localities with $\mathrm{Ng} \geq 15$ only

since the initial colonization. In many cases, reduced genetic diversity may persist for thousand of generations following a founder event (Hewitt 2000). Such reductions are of particular concern, especially in threatened and endangered species, because reductions in genetic diversity may limit the ability of populations to respond to environmental change (Willi and Hoffmann 2009). For example, clonal populations of reef corals may be more 
Table 5 S. hystrix locality statistics

\begin{tabular}{|c|c|c|c|c|c|c|c|}
\hline Locality & $\mathrm{N}$ & $\mathrm{Ng}$ & $\mathrm{Hz}$ & $\mathrm{Hz} \mathrm{SD}$ & $M$ & $M \mathrm{SD}$ & BAPS cluster \\
\hline Pulau Weh & 25 & 23 & 0.51 & 0.05 & 0.81 & 0.28 & 18 \\
\hline Anak Krakatau & 22 & 19 & 0.37 & 0.1 & 0.64 & 0.26 & 2 \\
\hline Rakata (Krakatau Besar) & 18 & 14 & NA & NA & NA & NA & NA \\
\hline Sangiang & 24 & 23 & 0.43 & 0.12 & 0.66 & 0.28 & 2 \\
\hline Sebesi & 9 & 4 & NA & NA & NA & NA & NA \\
\hline Sebuku 1 & 24 & 23 & 0.44 & 0.11 & 0.58 & 0.23 & 2 \\
\hline Sebuku 2 & 25 & 15 & 0.41 & 0.09 & 0.71 & 0.32 & 2 \\
\hline Alam Kotok & 20 & 20 & 0.41 & 0.09 & 0.67 & 0.34 & 2 \\
\hline Belat & 21 & 20 & 0.46 & 0.11 & 0.73 & 0.30 & 2 \\
\hline Karang Congkak & 21 & 20 & 0.41 & 0.14 & 0.67 & 0.31 & 2 \\
\hline Pramuka & 23 & 21 & 0.41 & 0.11 & 0.72 & 0.30 & 2 \\
\hline Pulau Pari & 38 & 38 & 0.53 & 0.11 & 0.5 & 0.18 & 2 \\
\hline Semak Daun & 21 & 20 & 0.5 & 0.1 & 0.58 & 0.23 & 2 \\
\hline Pemuteran & 41 & 41 & 0.41 & 0.11 & 0.63 & 0.3 & 1 \\
\hline Napoleon Reef & 21 & 21 & 0.46 & 0.12 & 0.59 & 0.32 & 1 \\
\hline Seraya & 20 & 18 & 0.42 & 0.08 & 0.64 & 0.36 & 8 \\
\hline Gili Trawangan & 12 & 12 & NA & NA & NA & NA & NA \\
\hline Kukusan & 1 & 1 & NA & NA & NA & NA & NA \\
\hline Bone Batang & 24 & 21 & 0.55 & 0.11 & 0.38 & 0.16 & 6 \\
\hline Gusung, Selayar & 23 & 21 & 0.54 & 0.1 & 0.70 & 0.31 & 9 \\
\hline Sebayur & 17 & 17 & 0.57 & 0.1 & 0.61 & 0.31 & 12 \\
\hline Batu Gosok & 12 & 12 & NA & NA & NA & NA & NA \\
\hline Murex House Reef (Bangka) & 34 & 34 & 0.68 & 0.07 & 0.70 & 0.29 & 10 \\
\hline Nudi Retreat & 36 & 28 & 0.64 & 0.12 & 0.82 & 0.16 & 3 \\
\hline Tanjung Pisok & 5 & 5 & NA & NA & NA & NA & NA \\
\hline Pulau Doi & 20 & 19 & 0.53 & 0.11 & 0.78 & 0.30 & 3 \\
\hline Jerewai & 22 & 21 & 0.44 & 0.1 & 0.79 & 0.25 & 14 \\
\hline Tidore & 28 & 15 & 0.27 & 0.1 & 0.58 & 0.33 & 7 \\
\hline Tonuu & 20 & 17 & 0.45 & 0.1 & 0.64 & 0.34 & 15 \\
\hline Alyui & 19 & 17 & 0.53 & 0.1 & 0.61 & 0.24 & 16 \\
\hline Gam & 1 & 1 & NA & NA & NA & NA & NA \\
\hline Jefman & 22 & 17 & 0.41 & 0.13 & 0.66 & 0.27 & 17 \\
\hline Kri & 9 & 9 & NA & NA & NA & NA & NA \\
\hline Mayalibit & 20 & 20 & 0.38 & 0.09 & 0.49 & 0.30 & 19 \\
\hline Adoki & 12 & 12 & NA & NA & NA & NA & NA \\
\hline Owi & 9 & 8 & NA & NA & NA & NA & NA \\
\hline Rasbar & 4 & 4 & NA & NA & NA & NA & NA \\
\hline Ambai & 22 & 20 & 0.45 & 0.13 & 0.72 & 0.28 & 4 \\
\hline Lemon & 20 & 20 & 0.5 & 0.11 & 0.67 & 0.31 & 13 \\
\hline Rumberpon & 19 & 19 & 0.42 & 0.1 & 0.79 & 0.31 & 5 \\
\hline Namatote & 21 & 21 & 0.41 & 0.11 & 0.81 & 0.28 & 20 \\
\hline Mommon & 18 & 18 & 0.56 & 0.1 & 0.70 & 0.32 & 11 \\
\hline
\end{tabular}

Shown for each locality are the number of samples taken $(\mathrm{N})$, the number of unique genotypes observed $(\mathrm{Ng})$, Nei's unbiased gene diversity $(\mathrm{Hz})$ and standard deviation, Garza-Williams statistic value $(M)$ and standard deviation, and the cluster to which each population was assigned by BAPS. Conditional posterior probabilities of assignments were all $\geq 0.99$. Results are presented for localities with $\mathrm{Ng} \geq 15$ only

susceptible to bleaching if the most common clone is thermally sensitive (Edmunds 1994). However, this is probably not the pattern observed in Krakatau. Coral genetic diversity in this very new habitat is comparable and, in the case of $P$. damicornis, higher than other localities in Indonesia. These observations show that it is 
Fig. 2 a $P$. damicornis BAPS cluster assignments with closeup view of the Krakatau region. Numbers indicate the cluster to which each study site was assigned, given in Table 4 . b $S$. hystrix BAPS cluster assignments with close-up view of the Krakatau region.

Numbers indicate the cluster to which each study site was assigned, given in Table 5
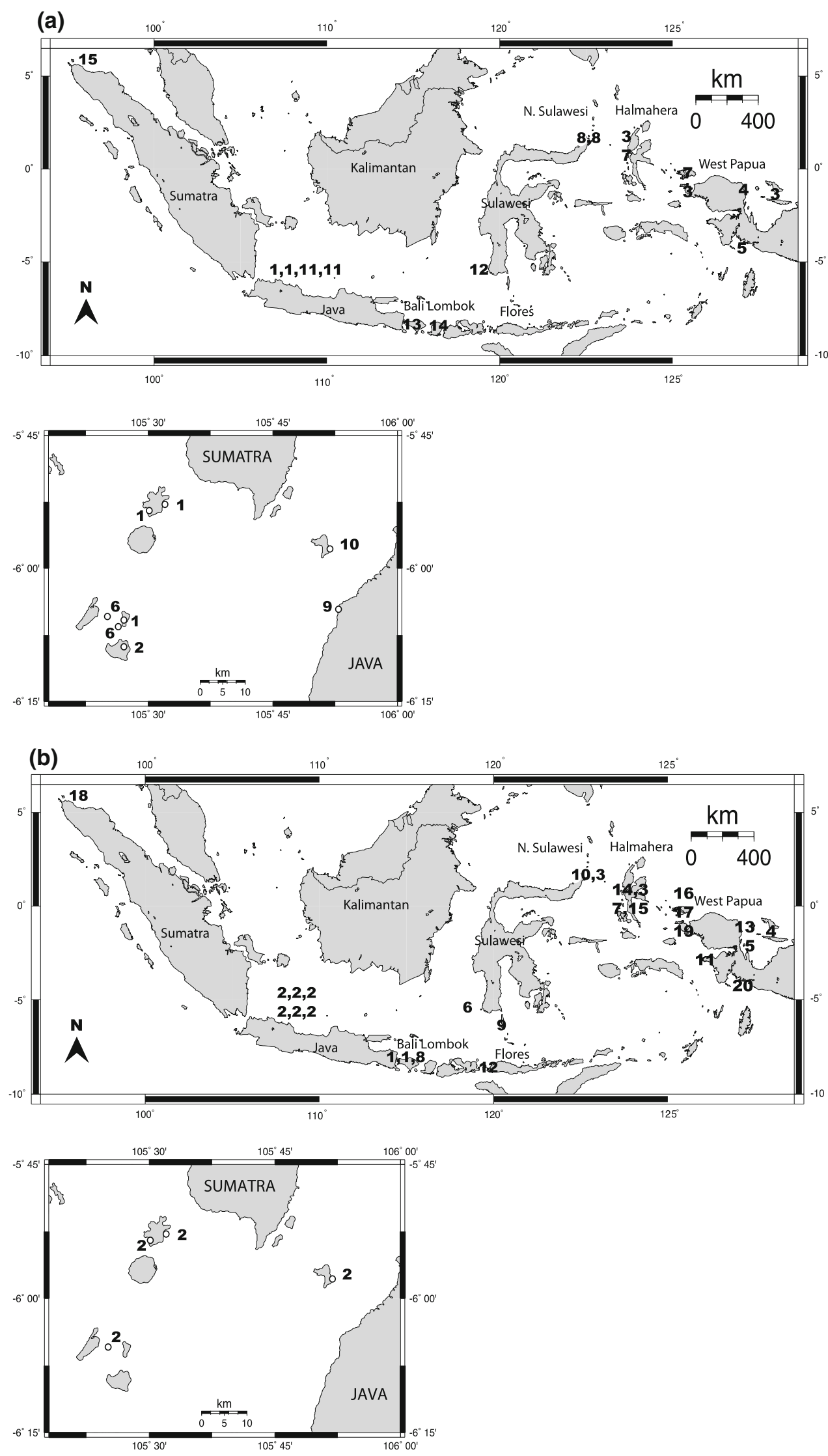
Table 6 P. damicornis: Pairwise $F_{\text {st }}$ among regions

\begin{tabular}{|c|c|c|c|c|c|c|c|c|c|c|c|c|c|}
\hline & \# & 1 & 2 & 3 & 4 & 5 & 6 & 7 & 8 & 9 & 10 & 11 & 12 \\
\hline Aceh & 1 & & & & & & & & & & & & \\
\hline Krakatau & 2 & 0.00 & & & & & & & & & & & \\
\hline Pulau Seribu & 3 & 0.00 & $\mathbf{0 . 0 3}$ & & & & & & & & & & \\
\hline Bali & 4 & 0.00 & 0.03 & 0.04 & & & & & & & & & \\
\hline Lombok & 5 & 0.00 & 0.04 & 0.05 & 0.00 & & & & & & & & \\
\hline Makassar & 6 & 0.00 & 0.10 & 0.11 & 0.05 & 0.06 & & & & & & & \\
\hline Northern Sulawesi & 7 & 0.00 & 0.04 & 0.03 & 0.03 & 0.06 & 0.13 & & & & & & \\
\hline Halmahera & 8 & 0.04 & 0.06 & 0.05 & 0.01 & 0.05 & 0.03 & 0.02 & & & & & \\
\hline Raja Ampat & 9 & 0.00 & 0.05 & 0.02 & 0.02 & 0.09 & 0.10 & 0.05 & 0.00 & & & & \\
\hline Biak & 10 & 0.00 & 0.01 & 0.00 & 0.00 & 0.07 & 0.07 & 0.04 & 0.00 & 0.00 & & & \\
\hline Yapen & 11 & 0.00 & 0.16 & 0.13 & 0.06 & 0.18 & 0.08 & 0.13 & 0.00 & 0.06 & $\mathbf{0 . 0 3}$ & & \\
\hline Manokwari & 12 & 0.00 & 0.10 & 0.08 & 0.12 & 0.21 & 0.21 & 0.10 & 0.09 & 0.11 & 0.08 & 0.21 & \\
\hline Kaimana & 13 & 0.00 & 0.04 & 0.02 & 0.00 & 0.11 & 0.10 & 0.03 & 0.00 & 0.02 & 0.01 & 0.11 & 0.09 \\
\hline
\end{tabular}

Bold values indicate significance at $p=0.05$ level after FDR correction

Table 7 S. hystrix: Pairwise $F_{\text {st }}$ among sub-regions

\begin{tabular}{|c|c|c|c|c|c|c|c|c|c|c|c|c|c|c|c|c|}
\hline & $\#$ & 1 & 2 & 3 & 4 & 5 & 6 & 7 & 8 & 9 & 10 & 11 & 12 & 13 & 14 & 15 \\
\hline Aceh & 1 & & & & & & & & & & & & & & & \\
\hline Krakatau & 2 & 0.22 & & & & & & & & & & & & & & \\
\hline Pulau Seribu & 3 & 0.22 & 0.02 & & & & & & & & & & & & & \\
\hline Bali & 4 & 0.22 & 0.04 & 0.04 & & & & & & & & & & & & \\
\hline Flores & 5 & 0.24 & 0.22 & 0.19 & 0.13 & & & & & & & & & & & \\
\hline Selayar & 6 & 0.28 & 0.13 & 0.09 & 0.12 & 0.12 & & & & & & & & & & \\
\hline Makassar & 7 & 0.26 & 0.22 & 0.19 & 0.13 & 0.10 & 0.14 & & & & & & & & & \\
\hline Northern Sulawesi & 8 & 0.14 & 0.10 & 0.10 & 0.05 & 0.10 & 0.08 & 0.13 & & & & & & & & \\
\hline Halmahera & 9 & 0.13 & 0.12 & 0.11 & 0.07 & 0.11 & 0.11 & 0.15 & 0.07 & & & & & & & \\
\hline Raja Ampat & 10 & 0.17 & 0.21 & 0.18 & 0.15 & 0.11 & 0.09 & 0.15 & 0.08 & 0.10 & & & & & & \\
\hline Biak & 11 & 0.20 & 0.20 & 0.18 & 0.15 & 0.11 & 0.09 & 0.16 & 0.07 & 0.12 & 0.09 & & & & & \\
\hline Yapen & 12 & 0.32 & 0.35 & 0.32 & 0.33 & 0.28 & 0.32 & 0.35 & 0.23 & 0.21 & 0.19 & 0.21 & & & & \\
\hline Manokwari & 13 & 0.31 & 0.36 & 0.33 & 0.31 & 0.25 & 0.28 & 0.31 & 0.23 & 0.21 & 0.17 & 0.19 & 0.14 & & & \\
\hline Teluk Cenderawasih & 14 & 0.3 & 0.29 & 0.27 & 0.25 & 0.27 & 0.32 & 0.29 & 0.18 & 0.19 & 0.15 & 0.18 & 0.13 & 0.14 & & \\
\hline Kaimana & 15 & 0.37 & 0.34 & 0.33 & 0.27 & 0.27 & 0.35 & 0.22 & 0.20 & 0.26 & 0.24 & 0.26 & 0.46 & 0.42 & 0.43 & \\
\hline Fakfak & 16 & 0.18 & 0.13 & 0.13 & 0.06 & 0.13 & 0.16 & 0.15 & 0.06 & 0.06 & 0.12 & 0.11 & 0.28 & 0.27 & 0.29 & 0.19 \\
\hline
\end{tabular}

Bold values indicate significance at $p=0.05$ level after FDR correction (All values were significant)

possible for coral reef animals to re-establish and recover genetic diversity in a matter of decades and centuries rather than millennia.

Comparison between the two species

Previous research into the population genetic patterns of corals (Ayre and Hughes 2004) leads one to expect faster recovery and higher genetic diversity in $P$. damicornis relative to $S$. hystrix. This hypothesis was also based on an unusually long larval life span in $P$. damicornis (Richmond 1987), possibly leading to dispersal from varied and distant sources. Although a direct comparison to larval life span in S. hystrix is not available, these genetic patterns support previous genetic studies, that larval dispersal may be more limited in S. hystrix.

A difference in genetic diversity in Krakatau between the two species is clear, but probably does not indicate a difference in the degree of recovery. In Krakatau the genetic diversity $(\mathrm{Hz})$ of $P$. damicornis (mean $\mathrm{Hz}=0.55$ ) is significantly higher than that of $S$. hystrix (mean $\mathrm{Hz}=0.41)$. While this might be taken to indicate more rapid recovery in $P$. damicornis due to higher gene flow, genetic diversity is typically higher in $P$. damicornis than 
Table 8 P. damicornis immigration matrix

\begin{tabular}{|c|c|c|c|c|c|c|c|c|c|c|c|c|c|c|}
\hline & & 1 & 2 & 3 & 4 & 5 & 6 & 7 & 8 & 9 & 10 & 11 & 12 & 13 \\
\hline Aceh & 1 & 0.69 & 0.00 & 0.00 & 0.00 & 0.01 & 0.00 & 0.00 & 0.00 & 0.00 & 0.01 & 0.00 & 0.00 & 0.00 \\
\hline Krakatau & 2 & $\underline{0.07}$ & 0.96 & 0.07 & 0.01 & $\underline{0.09}$ & 0.00 & 0.00 & 0.01 & 0.01 & 0.01 & 0.00 & 0.01 & 0.01 \\
\hline Pulau Seribu & 3 & 0.04 & 0.01 & 0.89 & 0.01 & 0.01 & 0.00 & 0.00 & 0.02 & 0.00 & 0.01 & 0.00 & 0.01 & 0.01 \\
\hline Bali & 4 & 0.01 & 0.01 & 0.00 & 0.81 & $\underline{0.05}$ & 0.00 & 0.00 & 0.00 & 0.00 & 0.01 & 0.00 & 0.00 & 0.02 \\
\hline Lombok & 5 & 0.01 & 0.00 & 0.00 & 0.00 & 0.68 & 0.00 & 0.00 & 0.00 & 0.00 & 0.01 & 0.00 & 0.00 & 0.00 \\
\hline Makassar & 6 & 0.01 & 0.00 & 0.00 & 0.01 & $\underline{0.08}$ & 0.99 & 0.00 & 0.01 & 0.00 & $\underline{0.05}$ & 0.00 & 0.00 & 0.02 \\
\hline Northern Sulawesi & 7 & 0.02 & 0.01 & 0.01 & $\underline{0.05}$ & 0.04 & 0.00 & 0.99 & 0.01 & $\underline{0.06}$ & $\underline{0.02}$ & 0.00 & 0.00 & $\underline{0.02}$ \\
\hline Halmahera & 8 & 0.01 & 0.00 & 0.00 & 0.00 & 0.01 & 0.00 & 0.00 & 0.67 & 0.00 & 0.01 & 0.00 & 0.01 & 0.00 \\
\hline Raja Ampat & 9 & $\underline{0.07}$ & 0.00 & 0.00 & 0.01 & 0.01 & 0.00 & 0.00 & $\underline{0.11}$ & 0.82 & $\underline{0.07}$ & 0.01 & 0.00 & 0.01 \\
\hline Biak & 10 & 0.01 & 0.00 & 0.00 & 0.00 & 0.01 & 0.00 & 0.00 & 0.00 & 0.00 & 0.68 & 0.00 & 0.00 & 0.00 \\
\hline Yapen & 11 & 0.02 & 0.01 & 0.01 & $\underline{0.08}$ & 0.01 & 0.00 & 0.00 & $\underline{0.14}$ & 0.09 & 0.07 & 0.96 & 0.01 & 0.00 \\
\hline Manokwari & 12 & 0.07 & 0.00 & 0.01 & 0.02 & 0.01 & 0.00 & 0.00 & 0.01 & 0.00 & 0.06 & 0.00 & 0.95 & 0.22 \\
\hline Kaimana & 13 & 0.01 & 0.00 & 0.00 & 0.00 & 0.01 & 0.00 & 0.00 & 0.00 & 0.00 & 0.01 & 0.00 & 0.00 & 0.68 \\
\hline
\end{tabular}

All standard deviation values are $\leq 0.05$. Bold values indicate self-recruitment rates for each region. Underlined values highlight immigration rates of $\geq 5 \%$

Table 9 S. hystrix immigration matrix

\begin{tabular}{llllllllllllllllll}
\hline & $\#$ & 1 & 2 & 3 & 4 & 5 & 6 & 7 & 8 & 9 & 10 & 11 & 12 & 13 & 14 & 15 & 16 \\
\hline Aceh & 1 & $\mathbf{0 . 9 9}$ & 0.00 & 0.00 & 0.00 & 0.00 & 0.01 & 0.00 & 0.04 & 0.00 & 0.00 & 0.00 & 0.00 & 0.00 & 0.00 & 0.00 & 0.01 \\
Krakatau & 2 & 0.00 & $\mathbf{0 . 7 5}$ & 0.00 & 0.00 & 0.01 & 0.01 & 0.00 & 0.00 & 0.01 & 0.00 & 0.12 & 0.00 & 0.01 & 0.00 & 0.00 & 0.01 \\
Pulau Seribu & 3 & 0.00 & $\underline{0.23}$ & $\mathbf{0 . 9 9}$ & 0.00 & 0.00 & $\underline{0.07}$ & 0.00 & 0.00 & $\underline{0.18}$ & 0.00 & 0.00 & 0.00 & 0.01 & 0.00 & 0.00 & 0.01 \\
Bali & 4 & 0.00 & 0.00 & 0.00 & $\mathbf{0 . 8 5}$ & 0.01 & 0.01 & 0.05 & 0.00 & 0.00 & 0.00 & 0.01 & 0.00 & 0.03 & 0.00 & 0.00 & 0.01 \\
Flores & 5 & 0.00 & 0.00 & 0.00 & 0.00 & $\mathbf{0 . 9 8}$ & 0.01 & 0.00 & 0.00 & 0.00 & 0.00 & 0.04 & 0.00 & 0.00 & 0.00 & 0.00 & 0.01 \\
Selayar & 6 & 0.00 & 0.00 & 0.00 & 0.00 & 0.00 & $\mathbf{0 . 6 8}$ & 0.01 & 0.00 & 0.00 & 0.00 & 0.00 & 0.00 & 0.00 & 0.00 & 0.00 & 0.01 \\
Makassar & 7 & 0.00 & 0.00 & 0.00 & 0.00 & 0.01 & 0.15 & $\mathbf{0 . 9 0}$ & 0.00 & 0.00 & 0.00 & 0.00 & 0.00 & 0.00 & 0.00 & 0.00 & 0.03 \\
Northern Sulawesi & 8 & 0.00 & 0.00 & 0.00 & 0.00 & 0.01 & 0.01 & 0.01 & $\mathbf{0 . 8 7}$ & 0.00 & 0.00 & 0.00 & 0.00 & 0.00 & 0.00 & 0.00 & 0.01 \\
Halmahera & 9 & 0.00 & 0.00 & 0.00 & 0.12 & 0.00 & 0.01 & 0.01 & 0.06 & $\mathbf{0 . 7 3}$ & 0.00 & 0.00 & 0.00 & 0.00 & 0.00 & 0.00 & 0.10 \\
Raja Ampat & 10 & 0.00 & 0.00 & 0.00 & 0.00 & 0.01 & 0.00 & 0.00 & 0.01 & 0.07 & $\mathbf{0 . 9 9}$ & 0.00 & 0.00 & 0.00 & 0.00 & 0.00 & 0.01 \\
Biak & 11 & 0.00 & 0.00 & 0.00 & 0.00 & 0.01 & 0.00 & 0.00 & 0.00 & 0.00 & 0.00 & $\mathbf{0 . 7 9}$ & 0.00 & 0.00 & 0.00 & 0.00 & 0.01 \\
Yapen & 12 & 0.00 & 0.00 & 0.00 & 0.00 & 0.00 & 0.00 & 0.00 & 0.00 & 0.00 & 0.00 & 0.00 & $\mathbf{0 . 9 9}$ & 0.00 & 0.00 & 0.00 & 0.01 \\
Manokwari & 13 & 0.00 & 0.00 & 0.00 & 0.00 & 0.01 & 0.01 & 0.00 & 0.00 & 0.00 & 0.00 & 0.00 & 0.00 & $\mathbf{0 . 9 2}$ & 0.00 & 0.00 & 0.01 \\
Teluk Cenderawasih & 14 & 0.00 & 0.00 & 0.00 & 0.00 & 0.00 & 0.01 & 0.00 & 0.00 & 0.00 & 0.00 & 0.00 & 0.00 & 0.00 & $\mathbf{0 . 9 8}$ & 0.00 & 0.01 \\
Kaimana & 15 & 0.00 & 0.00 & 0.00 & 0.00 & 0.01 & 0.00 & 0.00 & 0.00 & 0.00 & 0.00 & 0.00 & 0.00 & 0.00 & 0.00 & $\mathbf{0 . 9 8}$ & 0.11 \\
Fakfak & 16 & 0.00 & 0.00 & 0.00 & 0.00 & 0.01 & 0.00 & 0.00 & 0.00 & 0.00 & 0.00 & 0.00 & 0.00 & 0.00 & 0.00 & 0.00 & $\mathbf{0 . 6 8}$ \\
\hline
\end{tabular}

All standard deviation values are $\leq 0.05$. Bold values indicate self-recruitment rates for each region. Underlined values highlight immigration rates of $\geq 5 \%$

in S. hystrix throughout Indonesia suggesting that the differences observed in Krakatau are typical for Indonesian reefs. Considering this, recovery relative to the rest of Indonesia has largely occurred for both species.

Identifying larval sources for the colonization of Krakatau

The observed settlement and growth of reef corals less than 10 years following Krakatau's destruction (Sluiter 1890) indicates that dispersal to the Krakatau region from outside regions occurred almost immediately following the complete destruction of the coral reef biota. Coral larvae typically settle shortly after release meaning that, regardless of dispersal potentials, actual mean dispersal distance is very low (Sammarco and Andrews 1989; Isomura and Nishihira 2001). Results from pairwise gene flow estimates and the Bayesian assignment method implemented in BAPS indicate that $P$. damicornis populations in Krakatau have been colonized primarily by immigration from the closest reef 
system in Pulau Seribu, but supplemented from larval sources throughout the Indonesian Archipelago and possibly unsampled regions elsewhere. The rapid recovery of genetic diversity on Krakatau has also been observed for the mantis shrimp, Haptosquilla pulchella where larval sources were largely dominated by localities in the Java Sea (Barber et al. 2002). Similarly for $P$. damicornis, Rakata Kecil, Sebuku 1 and Sebuku 2 from Krakatau form one genetic cluster (Cluster \#1) that also includes two sites from Pulau Seribu (Alam Kotok and Semak Daun), suggesting that Pulau Seribu is the most likely source population for these reefs. This inference is supported most strikingly by pairwise $F_{\text {st }}$ estimates which are lower between Krakatau and Pulau Seribu than between Krakatau and any other region with only two exceptions. First, there was no significant $F_{\text {st }}$ between Krakatau and Pulau Weh in Aceh indicating that northern Sumatra may serve as an additional source population. Second, pairwise $F_{\text {st }}$ was not significant between Krakatau and Biak; however, this is not likely due to connectivity given the geographic distance between the two regions. Pairwise $F_{\text {st }}$ estimates also identify Bali as a possible source for the initial colonization of $P$. damicornis populations in Krakatau, supporting the hypothesis that the Java Sea was a major larval source for the recovery of Krakatau. In addition, Anak Krakatau and Karang Serang cluster only with each other in Cluster \#6 while, Sangiang, Anyer, and Rakata each clusters alone suggesting that not all larval sources have been sampled.

Similarly, larval sources for $S$. hystrix populations in Krakatau are dominated by nearby, upstream Java Sea populations in Pulau Seribu and Bali. Sangiang, Sebuku 1, Sebuku 2, and Anak Krakatau cluster with all populations in Pulau Seribu to form one genetic cluster that occurs nowhere else in Indonesia (Table 5). In addition, the lowest pairwise $F_{\text {st }}$ values observed anywhere in Indonesia in this species are between Krakatau and Pulau Seribu $\left(F_{\mathrm{st}}=0.02\right)$, between Krakatau and Bali $\left(F_{\mathrm{st}}=0.04\right)$, and between Bali and Pulau Seribu $\left(F_{\mathrm{st}}=0.04\right)$. These results are further supported by the migration estimates from BayesAss+, which identifies 23\% of the corals in Krakatau as migrants from Pulau Seribu. Taken together, these data suggest that these $S$. hystrix populations most likely originated via the immigration of coral larvae from the Java Sea. This is reasonable considering that surface currents in the Sunda Strait flow in a southwesterly direction from the Java Sea toward the Indian Ocean throughout the year (Wyrtki 1961). This also reinforces previous observations that populations of this extremely philopatric species typically depend on nearby sources of larvae for recovery (Underwood et al. 2007).

Long distance dispersal serves as a means by which coral populations can be founded and maintained over evolutionary time. Because the islands of Krakatau are geographically isolated from adjacent source populations, dispersal is likely to have initially occurred from nearby, unaffected reefs. Pairwise $F_{\text {st }}$ estimates indicate connectivity between Krakatau and Pulau Seribu support the original hypothesis. The assignment test implemented by BayesAss +, however, indicates not only that the Krakatau region is now predominantly self-seeding, but may also be serving as a larval source. Immigration estimates that identify Krakatau as connected to more distant sites upstream, including its function as a larval source, seem unlikely because of sea surface currents which flow from the Java Sea toward the Indian Ocean via the Sunda Strait. Nevertheless, immigration estimates indicating dispersal from Krakatau to Pulau Seribu and Biak, for example, may indicate unusual dispersal events mediated by pumicewhich is very common in the Krakatau region (personal observation) - or another rafting material (Jokiel 1984) during periods of atypical sea surface circulation. Connectivity over great distances has also been observed among East African P. damicornis populations while adjacent sites were also found to be genetically distinct (Souter et al. 2009). Coupled genetic, demographic, and physical oceanographic models may help resolve some of the apparent discontinuities between genetics and geography in coral population genetic studies (Galindo et al. 2006).

\section{Conservation implications}

The volcanic eruption and virtually instantaneous destruction of the coral reef ecosystems of Krakatau was a highly unusual event. However, many coral reef ecosystems throughout the world are now experiencing rapid and nearcomplete degradation. Mass mortalities of coral populations are projected to become increasingly common in the near future (McClanahan 2002; Gardner et al. 2003). Threats to coral reef ecosystems are well documented (Burke et al. 1997; Wilkinson 2004; reefsatrisk.wri.org); however, additional scientific data are still needed for their effective conservation. Connectivity has been listed as one of the most critical gaps in scientific knowledge needed for marine conservation (Sale et al. 2005). This is due largely to our need to accurately predict how recovery can occur following extreme (or even chronic) disturbance events. It is important to identify those populations that will serve as sources to areas that are likely to experience drastic population decline in the near future such as climate changeinduced coral bleaching and mortality (Underwood et al. 2007) and understand how remote coral reefs and MPAs may serve as larval sources and sinks (McClanahan et al. 2005; Miller and Ayre 2008). In the case study presented here, it was the availability of larvae in Pulau Seribu and Bali, and the dispersal corridor in the Java Sea, that served to repopulate coral reefs in the Krakatau region. 
The protection of potential source populations may prove critical in the recovery of degraded coral populations. However, the notion that protected areas can serve as sources of larvae and adults to surrounding areas, called the "spillover effect" has only been demonstrated in a limited number of cases, and typically only apply to adult fish and not their larvae (Palumbi 2004; Alcala et al. 2005; Ashworth and Ormond 2005; Sanchirico et al. 2006). Due to the highly self-seeding nature of coral populations and many marine ecosystems, external sources of larvae may not sustain downstream populations of coral reef animals over significant periods of time (Cowen et al. 2006). More likely, larval sources will serve to repopulate surrounding areas should disaster occur, and then only initially. This is highly dependent on the existence of healthy source populations. A complete recovery will depend on local sources of larvae in the form of healthy adult corals.

Acknowledgments We thank the Indonesian Institute of Sciences (LIPI), RISTEK, and all local authorities for research permission. Funding for coral collections was provided grants to PHB from NSF (OCE-0349177 and DEB-0338566) and Conservation International as well as a Pew Conservation Fellowship to Dr. Mark Erdmann. Laboratory work was supported by George Amato and Rob DeSalle at the Sackler Institute for Comparative Genomics at the American Museum of Natural History, and grants to ACB from the Tiffany \& Co. Foundation to the Wildlife Conservation Society and NSF (OCE0099301). CJS was supported by the Department of Ecology, Evolution, and Environmental Biology at Columbia University and an NSF GK-12 Teaching Fellowship administered though Columbia University's Chemistry Department. Benita Chick, Shinta Pardede, Eric Crandall, J.F. Bertrand, Yusuf Candika, and Jeannie Choi assisted with field work.

Open Access This article is distributed under the terms of the Creative Commons Attribution Noncommercial License which permits any noncommercial use, distribution, and reproduction in any medium, provided the original author(s) and source are credited.

\section{References}

Alcala AC, Russ GR, Maypa AP, Calumpong HP (2005) A long-term, spatially replicated experimental test of the effect of marine reserves on local fish yields. Can J Fish Aquat Sci 62:98-108

Arnaud-Haond S, Duarte CM, Alberto F, Serrao EA (2007) Standardizing methods to address clonality in population studies. Mol Ecol 16:5115-5139

Ashworth JS, Ormond RFG (2005) Effects of fishing pressure and trophic group on abundance and spillover across boundaries of a no-take zone. Biol Conserv 121:333-344

Atoda K (1947) The larva and postlarval development of the reefbuilding corals. I. Pocillopora damicornis cespitosa (Dana). The Science Reports of the Tohoku University, 4th Series 18:24-47

Atoda K (1951) The larva and postlarval development of the reefbuilding corals V. Seriatopora hystrix (Dana). The Science Reports of the Tohoku University Series 4:33-39
Ayre DJ, Dufty S (1994) Evidence for restricted gene flow in the viviparous coral Seriatopora hystrix on Australia Great Barrier Reef. Evolution 48:1183-1201

Ayre DJ, Hughes TP (2000) Genotypic diversity and gene flow in brooding and spawning corals along the Great Barrier Reef, Australia. Evolution 54:1590-1605

Ayre DJ, Hughes TP (2004) Climate change, genotypic diversity and gene flow in reef-building corals. Ecol Lett 7:273-278

Ayre DJ, Miller KJ (2004) Where do clonal coral larvae go? Adult genotypic diversity conflicts with reproductive effort in the brooding coral Pocillopora damicornis. Mar Ecol Prog Ser 277:95-105

Ayre DJ, Resing JM (1986) Sexual and asexual production of planulae in reef corals. Mar Biol 90:187-190

Ayre DJ, Hughes TP, Standish RS (1997) Genetic differentiation, reproductive mode, and gene flow in the brooding coral Pocillopora damicornis along the Great Barrier Reef, Australia. Mar Ecol Prog Ser 159:175-187

Barber PH, Palumbi SR, Erdmann MV, Moosa MK (2000) A marine Wallace's line? Nature 406:692-693

Barber PH, Moosa MK, Palumbi SR (2002) Rapid recovery of genetic diversity of stomatopod populations on Krakatau: temporal and spatial scales of marine larval dispersal. Proc R Soc Lond, B 269:1591-1597

Benjamini Y, Hochberg Y (1995) Controlling the false discovery rate: a practical and powerful approach to multiple testing. J Roy Stat Soc B 57:289-300

Burke L, Bryant D, McManus JW, Spalding M (1997) Reefs at risk: A map-based indicator of threats to the world's coral reefs. World Resources Institute, Washington, DC

Corander J, Marttinen P (2006) Bayesian identification of admixture events using multi-locus molecular markers. Mol Ecol 15:28332843

Corander J, Sirén J, Arjas E (2008) Bayesian spatial modeling of genetic population structure. Computation Stat 23:111-129

Cowen RK, Paris CB, Srinivasan A (2006) Scaling of connectivity in marine populations. Science 311:522-527

Edmunds PJ (1994) Evidence that reef-wide patterns of coral bleaching may be the result of the distribution of bleachingsusceptible clones. Mar Biol 121:137-142

Excoffier L, Laval G, Schneider S (2005) Arlequin ver. 3.0: an integrated software package for population genetics data analysis. Evol Bioinform Online 1:47-50

Falush D, Stephens M, Pritchard JK (2003) Inference of population structure using multilocus genotype data: linked loci and correlated allele frequencies. Genetics 164:1567-1587

Fan T-Y, Lin K-H, Kuo F-W, Soong K, Liu L-L, Fang L-S (2006) Diel patterns of larval release by five brooding scleractinian corals. Mar Ecol Prog Ser 321:133-142

Galindo HM, Olson DB, Palumbi SR (2006) Seascape genetics: A coupled oceanographic- genetic model predicts population structure of Caribbean corals. Curr Biol 16:1622-1626

Gardner TA, Cote IM, Gill JA, Grant A, Watkinson AR (2003) Longterm region-wide declines in Caribbean corals. Science 301:958960

Garza JC, Williamson EG (2001) Detection of reduction in population size using data from microsatellite loci. Mol Ecol 10:305-318

Glynn PW, Gassman NJ, Eakin CM, Cortes J, Smith DB, Guzman HM (1991) Reef coral reproduction in the eastern Pacific: Costa Rica, Panama, and Galapagos Islands (Ecuador). I. Pocilloporidae. Mar Biol 109:355-368

Grigg RW, Maragos JE (1974) Recolonization of hermatypic corals on submerged lava flows in Hawaii. Ecology 55:387-395

Harrison PL, Wallace CC (1990) Reproduction, dispersal and recruitment of scleractinian corals. In: Dubinsky $\mathrm{Z}$ (ed) 
Ecosystems of the world: coral reefs, vol 25. Elsevier, Amsterdam, pp 133-207

Hewitt G (2000) The genetic legacy of the Quaternary ice ages. Nature 405:907-913

Hughes TP (1984) Population dynamics based in individual size rather than age - a general model with a reef coral example. Am Nat 123:778-795

Isomura N, Nishihira M (2001) Size variation of planulae and its effect on the lifetime of planulae in three pocilloporid corals. Coral Reefs 20:309-315

Jokiel PL (1984) Long distance dispersal of reef corals by rafting. Coral Reefs 3:113-116

Karlson RH (2002) Dynamics of coral communities. Kluwer Academic Publishers, the Netherlands

Latch EK, Dharmarajan G, Glaubitz JC, Rhodes OE Jr (2006) Relative performance of Bayesian clustering software for inferring population substructure and individual assignment at low levels of population differentiation. Conserv Genet 7:295302

Lirman D (2000) Fragmentation in the branching coral Acropora palmata (Lamarck): growth, survivorship, and reproduction of colonies and fragments. J Exp Mar Biol Ecol 251:41-57

Magalon H, Samadi S, Richard M, Adjeroud M, Veuille M (2004) Development of coral and zooxanthella-specific microsatellites in three species of Pocillopora (Cnidaria, Scleractinia) from French Polynesia. Mol Ecol Notes 4:206-208

Mandeville C, Carey SHS, King J (1994) Paleomagnetic evidence for high temperature emplacement of the 1883 subaqueous pyroclastic flows from Krakatau volcano, Indonesia. J Geophys Res 99:9487-9504

McClanahan TR (2002) The near future of coral reefs. Environ Conserv 29:460-483

McClanahan TR, Maina J, Starger CJ, Herron-Perez P, Dusek E (2005) Detriments to post-bleaching recovery of corals. Coral Reefs 24:230-246

Meirmans PG, Van Tienderen PH (2004) GENOTYPE and GENODIVE: two programs for the analysis of genetic diversity of asexual organisms. Mol Ecol Notes 4:792-794

Miller K, Ayre D (2008) Protection of genetic diversity and maintenance of connectivity among reef corals within marine protected areas. Conserv Biol 22:1245-1254

Nei M (1987) Molecular evolutionary genetics. Columbia University Press, New York, New York, USA

Palumbi SR (2004) Marine reserves and ocean neighborhoods: the spatial scale of marine populations. Annu Rev Environ Resour 29:31-68

Park SDE (2001) Trypanotolerance in West African cattle and the population genetic effects of selection. Ph.D. thesis, University of Dublin

Peakall R, Smouse PE (2006) GENALEX 6: genetic analysis in Excel. Population genetic software for teaching and research. Mol Ecol Notes 6:288-295

Raymond M, Rousset F (1995) GENEPOP (Version 1.2): population genetic software for exact tests and ecumenicism. J Hered $86: 248-249$

Richmond RH (1987) Energetics, competency, and long-distance dispersal of planula larvae of the coral Pocillopora damicornis. Mar Biol 93:527-533

Sale PF, Cowen RK, Danilowicz BS, Jones GP, Kritzer JP, Lindeman KC, Planes S, Polunin NVC, Russ GR, Sadovy YJ, Steneck RS (2005) Critical science gaps impede use of no- take fishery reserves. Trends Ecol Evol 20:74-80

Sammarco PW, Andrews JC (1989) The Helix experiment: Different localized dispersal and recruitment patterns in Great Barrier Reef corals. Limnol Oceanogr 34:896-912
Sanchirico JN, Malvadkar U, Hastings A, Wilen JE (2006) When are no-take zones an economically optimal fishery management strategy? Ecol Appl 16:1643-1659

Sherman CDH (2008) Mating system variation in the hermaphroditic brooding coral, Seriatopora hystrix. Heredity 100:296-303

Sigurdsson H, Carey S, Mandeville C (1991) Submarine pyroclastic flows of the 1883 eruption of Krakatau volcano. Res Explor 7:310-327

Simkin T, Fiske RS (1983) Krakatau 1883: The volcanic eruption and its effects. Smithsonian Institution Press, Washington, DC

Slatkin M, Excoffier L (1996) Testing for linkage disequilibrium in genotypic data using the expectation-maximization algorithm. Heredity 76:377-383

Sluiter CP (1890) Einiges uber die Entstehung der Korallenriffen in der Javasee und Brantweibvai und uber neue Korllenbilung bei Krakatau. Natuurkundig Tijkschrift voor Nederlandsch Indie XLIX:360-380

Souter PB, Henriksson O, Olsson N, Grahn M (2009) Pattern of genetic structuring in the coral Pocillopora damicornis on reefs in East Africa. BMC Ecol 9:19

Starger CJ, Yeoh SSR, Dai CF, Baker AC, DeSalle R (2008) Ten polymorphic STR loci in the cosmopolitan reef coral Pocillopora damicornis. Mol Ecol Res 8:619-621

Stoddart JA (1984) Genetic differentiation amongst populations of the coral Pocillopora damicornis off Southwestern Australia. Coral Reefs 3:149-156

Thornton I (1996) Krakatau: The destruction and reassembly of an island ecosystem. Harvard University Press, Cambridge, Mass

Tomascik T, van Woesik R, Mah AJ (1996) Rapid coral colonization of a recent lava flow following a volcanic eruption, Banda Islands, Indonesia. Coral Reefs 15:169-175

Umbgrove JHF (1930) The end of Sluiter's coral reef at Krakatoa. Leidsche Geologische Mededeelingen 3:261-264

Underwood JN, Souter PB, Ballment ER, Lutz AH, van Oppen MJH (2006) Development of ten polymorphic microsatellite markers from herbicide bleached tissues of the brooding pocilloporid coral Seriatopora hystrix. Mol Ecol Notes 6:176-178

Underwood JN, Smith LD, van Oppen MJH, Gilmour JP (2007) Multiple scales of genetic connectivity in a brooding coral on isolated reefs following catastrophic bleaching. Mol Ecol 16:771-784

Van Oppen MJH, Lutz A, De'ath G, Peplow L, Kininmonth S (2008) Genetic traces of recent long-distance dispersal in a predominantly self-recruiting coral. PLoS ONE 3:e3401

Veron JEN (2000) Corals of the world. Australian Institute of Marine Science, Townsville

Villanueva RD, Yap HT, Montaño MNE (2008) Timing of planulation by pocilloporid corals in the northwestern Philippines. Mar Ecol Prog Ser 370:111-119

Walsh PS, Metzger DA, Huiguchi R (1991) Chelex ${ }^{\circledR} 100$ as a medium for simple extraction of DNA for PCR-based typing from forensic material. BioTechniques 10:506-513

Wilkinson C (2004) Status of coral reefs of the world: 2004. Australian Institute of Marine Science, Townsville, Australia

Willi Y, Hoffmann AA (2009) Demographic factors and genetic variation influence population persistence under environmental change. J Evol Biol 22:124-133

Wilson GA, Rannala B (2003) Bayesian inference of recent migration rates using multilocus genotypes. Genetics 163:1177-1191

Wyrtki K (1961) Physical oceanography of the southeast Asian waters. Scripps Institution of Oceanography Naga Report 2, La Jolla, CA

Zakai D, Levy O, Chadwick-Furman NE (2000) Experimental fragmentation reduces sexual reproductive output by the reefbuilding coral Pocillopora damicornis. Coral Reefs 19:185-188 